\title{
Review Article Electroencephalography in Mesial Temporal Lobe Epilepsy: A Review
}

\author{
Manouchehr Javidan $1,2,3$ \\ ${ }^{1}$ Division of Neurology, Department of Medicine, University of British Columbia, Vancouver, BC, Canada V5Z 1M9 \\ ${ }^{2}$ Neurophysiology Laboratory, Vancouver General Hospital, Vancouver, BC, Canada V5Z1M9 \\ ${ }^{3}$ Epilepsy Program, Vancouver General Hospital, Vancouver, BC, Canada V5Z 1M9
}

Correspondence should be addressed to Manouchehr Javidan, mjavidan@vch.ca

Received 12 October 2011; Revised 17 January 2012; Accepted 23 February 2012

Academic Editor: Seyed M. Mirsattari

Copyright (c) 2012 Manouchehr Javidan. This is an open access article distributed under the Creative Commons Attribution License, which permits unrestricted use, distribution, and reproduction in any medium, provided the original work is properly cited.

Electroencephalography (EEG) has an important role in the diagnosis and classification of epilepsy. It can provide information for predicting the response to antiseizure drugs and to identify the surgically remediable epilepsies. In temporal lobe epilepsy (TLE) seizures could originate in the medial or lateral neocortical temporal region, and many of these patients are refractory to medical treatment. However, majority of patients have had excellent results after surgery and this often relies on the EEG and magnetic resonance imaging (MRI) data in presurgical evaluation. If the scalp EEG data is insufficient or discordant, invasive EEG recording with placement of intracranial electrodes could identify the seizure focus prior to surgery. This paper highlights the general information regarding the use of EEG in epilepsy, EEG patterns resembling epileptiform discharges, and the interictal, ictal and postictal findings in mesial temporal lobe epilepsy using scalp and intracranial recordings prior to surgery. The utility of the automated seizure detection and computerized mathematical models for increasing yield of non-invasive localization is discussed. This paper also describes the sensitivity, specificity, and predictive value of EEG for seizure recurrence after withdrawal of medications following seizure freedom with medical and surgical therapy.

\section{Introduction}

The previous ILAE classification of epilepsy syndromes classified temporal lobe epilepsy under medial temporal lobe (mTLE) and lateral temporal lobe epilepsy (nTLE) [1]. In the revised classification, mTLE is under "distinctive constellations/electroclinical syndromes" with the most common pathological substrate being hippocampal sclerosis (HS) [2, $3]$.

mTLE-HS is the most common form of focal epilepsy. It has a distinct clinical presentation called "limbic seizure" resulting from epileptic activity within the limbic structures; however, they are not distinguishable from mTLE with other pathologies than HS. mTLE-HS also has specific electrographic features, neuroimaging, and pathological findings. The term hippocampal sclerosis denotes a specific pattern of cell loss mostly involving CA1 and hilar neurons and least the CA2 region [4]. Although the pathophysiology of this condition based on human surgical tissue and in animal studies is well known, the sequence of events resulting in HS is not well understood [5]. Hippocampal sclerosis can now be detected by high-resolution MRI in majority of patients $[6,7]$.

The incidence and prevalence of mTLE are not well known, and the available information regarding natural history is biased by those patients with refractory seizures who are referred to the epilepsy surgery centers. In one series, pathologic analysis of the hippocampal tissue revealed that up to $70 \%$ of patients with intractable temporal lobe epilepsy may have HS [4]. Two longitudinal outpatient studies addressed the prevalence of this condition. The French study included tertiary referrals, and the other was conducted at a primary care clinic in Glasgow $[8,9]$. In the former, half of patients had TLE. In both studies, about $25 \%$ of patients had hippocampal atrophy on MRI, and seizures were most refractory in this group. The Paris study reported 
seizure freedom in only $11 \%$ and $3 \%$ of those with dual pathology (HS and another MRI lesion) in the previous year. This report suggested that mTLE-HS may be the most common form and most medically refractory epilepsy [8].

The autosomal dominant familial form of mTLE has been described to have a more benign course $[10,11]$. The clinical and EEG manifestations of the familial form cannot be distinguished from the sporadic form. The patients with refractory seizures in this group similarly benefit from surgery [12].

The family history of epilepsy, history of prolonged febrile seizure or other insults such as trauma and infection in early life are typical of the syndrome of mTLE with HS [5]. The limbic seizure is often characterized by an aura followed by a dyscognitive state with variable degrees of impaired level of consciousness. The auras and seizure behaviour are fairly characteristic in most [13], however, in a subgroup of patients the clinical manifestations are atypical and make the diagnosis quite challenging. Examination is often normal except for memory deficits.

When the typical history is missing, the limbic seizure could have another pathological substrate or the epileptic abnormality projects to the mesial structures from the temporal neocortex or the close neighbouring structures such as orbitofrontal cortex, the insula or frontal/parietal operculum. These seizures could be clinically indistinguishable from mTLE-HS. The term temporal "plus" (T+) was introduced by Ryvlin and Kahane [14] to describe the form of seizures with a complex epileptogenic network. The ictal clinical presentation and scalp EEG assist in differentiating TLE from seizure originating in the neighbouring structures [15].

mTLE with HS is the most common refractory epilepsy in adults and has a poor prognosis with medical treatment. However, many of these patients have excellent response to surgery and removal of the epileptogenic zone. One prospective study found that surgery was clearly superior to medical therapy in this population of patients [16]. A large number of other studies have reported $60-80 \%$ of seizure freedom after the mesial temporal lobe resection [17-19]. In children, focal cortical dysplasia is more common pathological substrate than HS. Mittal et al. and Lerner et al. have reported the results of surgery for TLE in children $[20,21]$.

In presurgical evaluation of patients with TLE, the identification of seizure focus is the most important prerequisite for surgery. EEG in temporal lobe epilepsy has been recently reviewed [22, 23]. A detailed clinical assessment, interictal and ictal EEG findings with electroclinical correlation during continuous video/EEG monitoring, neuropsychological data, MRI, and sometimes single-photon emission computed tomography (SPECT)/positron emission tomography (PET) or magnetoencephalography (MEG) have been utilized for the identification of the epileptic focus. In more recent years significant advances in digital EEG, seizure detection technology, MRI, EEG/MRI superimposition, MR spectroscopy, and functional MRI (fMRI) have been very helpful in noninvasive localization of the seizure focus [24, 25]. Neuropsychological assessment and WADA for determining the language side and lateralized memory dysfunction aid in surgical decision [26]. Electrocorticography in the operating room can localize the active seizure focus and guide the surgical resection [27].

When the information obtained from the scalp EEG, MRI and neuropsychological assessment are insufficient or nonconcordant, or when further data is required, invasive intracranial EEG recording will be planned. Placement of subdural strip/grid or depth electrodes provides valuable information for localization of the seizure focus. The extent and location of electrode placement is designed to test the hypotheses regarding the possible ictal onset zones. The hypotheses are generated from clinical semiology, scalp EEG, neuropsychological and imaging data.

The focus of this paper is on the electroencephalographic aspects of mTLE with some attention to the other aspects of this syndrome related to EEG. The semiology of TLE, mTLE, nTLE, surgical treatment of TLE, and pediatric TLE are subjects of other papers in this special issue, and the readers are referred to those papers for further details.

\section{Method of This Review}

The MEDLINE literature search was performed for the EEG information and pertaining to all the aspects of the mesial temporal lobe epilepsy. The focus of this search was mainly on the EEG features of mTLE and detail of clinical features, neuroimaging, pathology and surgical results are not included. All the original articles and pertinent references in the several main reference textbooks on this topic were reviewed. Although there were no age limits, most of the information regarding the EEG data were obtained from the adult literature.

\section{Historical Aspects of EEG in Temporal Lobe Epilepsy}

A specific form of EEG characterized by rhythmic sharp waves and 6 per second waves was first described by Gibbs et al. in 1935 as the typical manifestation of psychomotor seizures [28]. Lewis is one of the pioneers who recorded EEG in patients with behavioural disorders and epilepsy. He was able to identify epileptogenic foci based on ictal and interictal discharges in 55 patients with epilepsy. Using bipolar EEG, he could also predict the location of the lesion within 2$3 \mathrm{~cm}$ in $85 \%$ of patients being evaluated for brain surgery for other lesions [29]. The use of EEG significantly improved the surgical results and led others to utilize EEG prior to surgery in 1930s and 1940s.

Lewis could distinguish the "seizure onset zone" from the "epileptogenic lesion" identified by the only available pneumoencephalogram and EEG soon became an important tool in classification, pathophysiology, localization, and surgical treatment of epilepsy [29].

The role of EEG in temporal lobe epilepsy (TLE) and epilepsy surgery was first described by Kennedy and Hill in 1958 [30]. Bailey and Gibbs in 1951 [31] were the pioneers who resected the anterior temporal lobe mainly based on the EEG evidence. Since the time of Jasper EEG has remained the main tool to guide surgery. In 1975, Engel reviewed 
the electrophysiological correlates of pathology and surgical results in temporal lobe epilepsy [32]. The role of EEG in epilepsy and the advancement of digital EEG and automatic spike and seizure detection have been reviewed elsewhere [33-35].

\section{Technical Considerations}

Electrode placement using the international ten-twenty system was first introduced in 1958 [36]. This system is now widely accepted and used as the standard method of measurement and application of electrodes for recording scalp EEG. Standard electrodes can detect only up to $58 \%$ of spikes [37]. Recently the original 10-20 electrode system and alphanumeric nomenclature were modified. Additional and more closely spaced electrodes allow better identification and localization of the epileptiform discharges in the subtemporal and other areas in patients with partial seizures and TLE [38].

Utilizing special electrodes such as nasopharyngeal, sphenoidal [39], anterior temporal (AT or T1, T2), and mandibular notch $(\mathrm{MN})$ electrodes have increased the yield of detecting epileptiform discharges in the mediobasal region [40]. Recently Wilkus et al. [41] evaluated and compared the ictal changes over different electrodes and reported equivalent results. Ives et al. [42] found the montages containing the sphenoidal electrodes detected the ictal onset usually by more than 5 seconds compared to the other montages and were able to detect ictal changes not apparent in the anterior posterior temporal montage.

Recently, using intracranial recording as gold standard, the sensitivity of the surface and sphenoidal electrodes was found to be comparable [43]. T1, T2, and MN electrode are able to record almost all the sphenoidal detected spikes. Nasopharyngeal electrodes are uncomfortable and have not been proven to be superior to the other electrode types and therefore are no longer widely used.

Activating procedures can increase the yield of detecting interictal epileptiform discharges. Hyperventilation is particularly useful for primary generalized epilepsies; however, it can also activate focal epileptiform discharges in up to $10 \%$ in partial epilepsies [44]. Photic simulation is useful for activation of generalized epileptiform discharges and has not been used for partial seizures. Several studies have documented that sleep deprivation can increase the chance of detecting epileptiform discharges in both partial and generalized epilepsies $[45,46]$. This is mainly due to the effect of sleep deprivation and not the sleep per se.

\section{The EEG Patterns Resembling Epileptiform Activity}

5.1. Benign EEG Variants. The distinction between interictal epileptiform discharges (IED) and benign EEG variants (EEG patterns with unknown aetiology) is at times challenging and poses difficulty for the EEG interpretation. These patterns have been extensively reviewed in the literature $[47,48]$. The waveforms that mimic epileptiform patterns of TLE are listed in Table 1.
TABLE 1: Benign EEG variants that could mimic epileptiform discharges.

Small sharp spikes of sleep (SSS or BETS)
Wicket spikes
Psychomotor variant (RMTD)
Mu rhythm
Subclinical rhythmic electrographic (theta) discharges in adults
(SREDA)

$B E T S$ are short-duration (less than 50 milliseconds) and low-amplitude (less than 50 microvolts) spikes. They occur during early sleep and are located in the temporal and frontal regions unilaterally or bilaterally but in most instances they are asymmetrical. They do not have a prominent aftercoming slow wave although they may have a small dip or single aftercoming slow wave component. Unlike the temporal epileptiform sharp waves, BETS do not distort the background and do not occur in trains. They often have a broader field than temporal epileptiform discharges and do not have clinical significance related to TLE [49].

Wicket spikes are single or trains of sharply contoured monophasic arciform waves similar to train of wicket-like activity that occur mainly during drowsiness and light sleep. When they occur as a single high-amplitude waveform, they could be mistaken for an abnormal temporal lobe epileptiform discharge. Wicket spikes do not have after coming slow wave component and do not distort the background [50].

Rhythmic mid-temporal discharges (RMTD) or psychomotor variant occur in early sleep. This is a $5-7 \mathrm{~Hz}$ semirhythmic waveform with a notched appearance lasting few to several seconds, and it is often unilateral and predominantly in the temporal regions. It has an abrupt onset and ending without evolution which can differentiate RMTD from buildup of ictal activity. There is no associated clinical symptom, and this pattern is not related to TLE [51].

$\mathrm{Mu}$ rhythm is a central rhythm with arch-like configuration with an alpha range $8-10 \mathrm{~Hz}$ frequency, which occurs more in adult and less in elderly. It blocks with movements of the contralateral limbs. If sharply contoured and high amplitude over a bone defect, it may be mistaken for epileptiform discharges [52].

Subclinical Rhythmic Electrographic Discharges in Adults (SREDA). This is an uncommon pattern mostly in people over the age of 50 years. It may occur during drowsiness or at rest. It contains theta delta frequencies evolving into a 5$7 \mathrm{~Hz}$ range maximum over the parietotemporal regions. It is usually bilateral, but it could be asymmetrical. SREDA may occur in a sharply contoured and single-waveform configuration, and it could be repetitive and evolving resembling subclinical seizures $[53,54]$.

5.2. Artifacts and Breech Rhythm. Different movements can cause artifacts on scalp EEG that could mimic epileptiform activity. These include chewing and swallowing movements, movements of the eyes, head or limbs, rubbing, tremor and 
scratching, that could cause rhythmic changes or spike-like discharges. Mechanical artifacts are often due to instability of electrode on the scalp and electrode wire or cable movement.

Scalp changes by trauma, defects by craniotomy and breech rhythm, electronic devices that generate alternating current fields such as ventilators or intravenous pumps and static changes by dripping intravenous fluids could cause artifacts resembling epileptiform spikes or sharp waves [55]. Therefore, spike like discharges only during sleep and those without a recognizable field and aftercoming slow waves should be considered nonepileptiform.

\section{Continuous Video/EEG Recordings (CV-EEG)}

The probability of capturing a seizure during a routine 20 30 minutes EEG in a patient with average of one seizure per week is reported to be about $1 \%$ [56]. CV-EEG recording is the ultimate technology in the analysis of clinical and subclinical seizures for the diagnosis of the seizure type and epileptic syndrome as well as differentiating epileptic from nonepileptic spells [57]. CV-EEG has also been helpful in the correct diagnosis of epilepsy in those patients with the misdiagnosis of non-epileptic seizures $[58,59]$. Other than increasing the likelihood of detecting interictal epileptiform activity, CV-EEG allows visual analysis of the seizures and simultaneous clinical and electrographic correlation helpful in presurgical evaluation. Although CV-EEG is mostly utilized in an inpatient setting, it is also used for shorter day recordings particularly in children with frequent seizures $[60,61]$. The information from CV-EEG along with imaging and neurophysiologic data guides the clinicians in surgical resection of the epileptogenic zone in temporal lobe surgery.

\section{Interictal Scalp EEG in Epilepsy}

The first question the clinician asks is whether the patient with paroxysmal events has epilepsy. Although this is primarily a clinical diagnosis, EEG plays an important role. The pattern of interictal EEG assists in determining the seizure type; however, different EEG patterns have different association with epilepsy, and therefore the sensitivity and specificity of EEG patterns remain unclear. EEG abnormalities could be in the form of interictal epileptiform discharges (IEDs), focal-slowing or periodic-lateralized epileptiform discharges (PLEDs) or diffuse changes such as frontal intermittent rhythmic delta activity (FIRDA). Only IEDs and PLEDs have sufficiently high association with epilepsy [55]. In focal epilepsies, the interictal spike is a guide for seizure focus localization along with clinical and imaging data. Table 2 highlights the features helping to identify IED. Although not inevitable, these features are highly correlated with epilepsy.

Interictal EEG documents the background cortical function during the nonseizure state. For example, generalized epileptiform discharges in the context of normal background activity are more characteristic of genetic epilepsies. The presence of frequent IED or significant background slowing could be associated with the patient's cognitive abnormality. Table 3 indicates some of the clinical uses of IEDs.
TABLe 2: The characteristics of interictal epileptiform discharges [55].

Paroxysmal with spiky configuration standing out from the background

Duration 70-200 msec for a sharp wave and 20-70 msec for a spike

Abrupt change in polarity

Surface negative polarity

Have a physiological field

TABle 3: The clinical information obtained by interictal epileptiform discharges [55].

\begin{abstract}
Diagnosis of epilepsy in paroxysmal events
Identification of the refractory epilepsies

The risk of seizure recurrence after a single unprovoked seizure Diagnosis of epileptic syndromes and predicting the prognosis Identifying the surgically remediable epilepsies Probability of seizure recurrence after medication withdrawal Predication of seizure recurrence after medication withdrawal following surgery
\end{abstract}

\subsection{Sensitivity and Specificity of Interictal Epileptiform} Discharges. EEG could direct toward a particular type of epilepsy and help in distinguishing among several possible syndromes in complex and confusing clinical situations. However, critical analysis of sensitivity, specificity, and positive predictive value of different EEG features is not possible [55].

7.1.1. Sensitivity. Three large studies of mainly adult patients reported a range of sensitivity of EEG in detecting IEDs. The initial EEG is positive for epileptiform discharges in $29-55 \%$, being more sensitive in generalized epilepsies [62]. Repeated EEGs and longer duration of recordings increase the yield to $80-90 \%$ [63]. EEG is persistently negative in about $8 \%$ of patients with epilepsy and higher proportions of patients with partial seizures have negative interictal EEG [64]. EEG has a lower yield, estimated between $12 \%$ and $50 \%$, in patients who had only one seizure or those who were well controlled and would undergo medication withdrawal $[65$, 66]. Hence, the interictal EEG has variable sensitivity, and its yield depends upon a number of factors listed in Table 4 [67-69]. Automatic detection methods have been of great assistance in spike detection during long-term monitoring of epileptic patients [70].

Antiepileptic drugs such as benzodiazepines, valproic acid, and barbiturates can suppress IEDs [71-73]. Other than magnetoencephalography (MEG), several different computerized techniques have improved the sensitivity of IED detection by EEG signals [74, 75].

The yield of interictal abnormality in TLE depends on different factors such as duration of recording and the state of the patient. One video/EEG study with a mean of 6.9 days of continuous monitoring showed that $19 \%$ of patients with recorded seizures had no interictal abnormality [64]. In our 
TABLE 4: Conditions that increase the yield of EEG detecting interictal epileptiform discharges.

In children and seizure starting in earlier life

Within 48 hours after a seizure

Certain epilepsy syndromes such as benign rolandic epilepsy,

Lennox Gastaut, and Landau Kleffner

Greater seizure frequency

Sleep and sleep deprivation

Serial EEGs

patient population with mTLE who underwent surgery after video/EEG monitoring and had good surgical outcome, CVEEG failed to reveal IED in 15 out of 171 patients $(8.7 \%)$ (unpublished data).

7.1.2. Specificity. IEDs are very uncommon in people without epilepsy. Studies have reported IED in $0.5 \%$ in adults and $1.9 \%$ to $3.5 \%$ of normal children without epilepsy. The interictal epileptiform discharges typical of partial epilepsy are very rare in people without partial epilepsy [76-78].

7.2. Positive Predictive Value of Interictal Epileptiform Discharges. This is calculated by the ratio of the number of subjects with epilepsy who have IED to all subjects with or without epilepsy who have IEDs. This ratio is dependent on the sensitivity and specificity of IEDs and more importantly the population under study. Therefore it could vary depending on the outpatient versus hospitalized patients [64].

7.3. Predictive Value of Interictal Epileptiform Discharges for Seizure Recurrence. Factors associated with persisting seizure freedom versus seizure recurrence after withdrawal of antiseizure drugs following surgery have been studied. Shinnar et al. in a prospective study followed 407 children who presented with a first unprovoked seizure followed for a mean of 6.3 years from the time of the first seizure. Children with first seizure and unidentified cause and a normal EEG had a 5-year recurrence risk of only $21 \%$ [79].

Another study analyzed the sensitivity, specificity, and positive and negative predictive values of EEG for seizure recurrence on 831 children in four eligible studies searched between 1980 and 1998. They reported a low sensitivity of $61 \%$ and specificity of $71 \%$ and concluded that EEG should not be obtained routinely after first unprovoked seizure in childhood [80].

Prospective and randomized studies as well as one metaanalysis in adult population have revealed that an abnormal EEG was associated with an increased risk of recurrence, but in some studies there was considerable variability in the results, and the epileptiform activity was not differentiated in most studies [81-83].

Tinuper et al. assessed the role of the EEG in predicting seizure recurrence in partial epilepsies. They found that the interictal EEG at the time of antiepileptic drug withdrawal did not predict recurrence, however, a worsening of the EEG after withdrawal was predictive of seizure recurrence [84].
7.4. The Value of EEG in Predicting Seizure Recurrence after Temporal Lobe Resection. Studies have reported contradictory results in the literature. However, collectively it seems that an abnormal EEG can predict the possibility of seizure recurrence after withdrawal of medications following surgery for temporal lobe epilepsy.

The outcome of discontinuation of anti-seizure medications was retrospectively studied in 210 consecutive patients who were rendered seizure-free after epilepsy surgery performed between 1989 and 1993. Medications were reduced in 96 and discontinued in 84 patients. The seizure recurrence rate after complete anti-seizure withdrawal was $14 \%$ and $36 \%$ at 2 and 5 years. In contrast, only 3\% and 7\% of the 30 patients who did not alter anti-seizure medications after surgery had recurrent seizures in the same time intervals. Intraoperative electrocorticography and postoperative EEG were not predictive of seizure outcome after anti-seizure drug withdrawal [85].

In another study serial EEGs at 3 months, and at 1, 2, and 3 years after anterior temporal lobectomy were analysed in 262 consecutive patients with mTLE-HS. EEG was considered abnormal when interictal epileptiform discharges (IED) were present. Anti-seizure medication withdrawal was attempted in all seizure-free patients. Favourable outcome was defined as freedom from seizures/auras during the entire follow-up period (outcome 1) and during terminal 1-year followup (outcome 2). Those with IED had 326 fold increase in the risk of seizure recurrence. The authors concluded that EEG after anterior temporal resection predicts seizure outcome and seizure recurrence following medication withdrawal. Serial EEGs predict outcome better than single EEG [86]. Several other studies have addressed the prediction of seizure recurrence after temporal lobe surgery $[87,88]$.

\section{Interictal Findings in mTLE: Scalp EEG Recording}

The interictal changes in mTLE could be in the form of nonepileptiform abnormalities, epileptiform discharges, or both.

\subsection{Non-Epileptiform}

8.1.1. Focal Dysrhythmia/Slowing. Scalp EEG may reveal a persistent or intermittent $4-7 \mathrm{~Hz}$ (Theta) or $1-3 \mathrm{~Hz}$ (delta activity) unilaterally or bilaterally in the temporal regions. This is a nonspecific finding in various conditions such as tumours, stroke, and HS, or it may not have a pathological substrate. If persistent, it is more consistent with structural abnormality. However, if neuroimaging does not reveal any pathological substrate, the intermittent slow waves in the temporal region are often due to interictal or postictal activity [89].

Lateralized focal or regional polymorphic delta activity is frequently found in TLE and is highly associated with temporal spiking. Koutroumanidis et al. correlated the interictal temporal delta activity in TLE with the pathology and surgical outcome. They reported lateralized slow activity 
in $66 \%$ of 141 patients who had temporal lobe resection for intractable partial seizures. The delta activity correlated well with the side of the temporal spikes. It provided additional information in 15\%, in whom EEG did not show lateralized interictal spikes. The authors concluded that in patients with TLE, whose MRI was either normal or suggestive of HS, interictal temporal slow activity had a lateralizing value similar to that of temporal spiking and was significantly associated with favourable surgical outcome [90].

In another study, $82 \%$ of patients with seizures exclusively from one temporal lobe had unilateral delta activity ipsilateral to the side of the ictal onset; this activity never falsely lateralized the seizure onset [91].

\subsubsection{Temporal Intermittent Rhythmic Delta Activity} (TIRDA). This term consists of trains of rhythmic delta activity lasting 4-20 seconds and is observed in up to $25 \%$ of patients with TLE who are being evaluated for surgery. TIRDA is more specific to TLE and often associated with epileptiform discharges [92, 93]. In one study, TIRDA was found in up to $90 \%$ of patients with MRI evidence of hippocampal atrophy and mTLE [94]. The authors concluded that delta activity lateralized to the side of the atrophy had accuracy equal to the spikes and reflected the epileptogenic process rather than the structural pathology. This specificity of TIRDA was also confirmed by Di Gennaro et al. [95]. Figure 1 demonstrates an example of TIRDA.

\subsection{Epileptiform}

8.2.1. Spikes and Sharp Waves: Interictal Epileptiform Discharges (IEDs). The typical epileptiform abnormality is the characteristic spike or sharp wave with negative polarity and is often followed by a slow wave (Figure 2). The anterior temporal spikes have maximum negativity over the temporal basal electrodes (F7, F8, T1, T2, and sphenoidal electrodes) $[96,97]$. In a temporal lobe surgical series, the anterior temporal spikes were present in up to $94 \%$ of 64 patients with mTLE [98]. Figure 2 demonstrates an example.

Computer field mapping technique has found that the maximum electronegativity of these spikes is anterior and inferior to the standard 10-20 electrode positions [99]. This field improves the sensitivity of the additional nonstandard electrodes. Furthermore, the analysis of the voltage topography revealed that the maximum region of electronegativity of these spikes was over the ipsilateral inferior temporal scalp associated with a more diffuse area of positivity over the contralateral central parietal scalp [100].

The precise anatomical generators of scalp and sphenoidal detected spikes have not yet been identified with certainty. Wilkus et al. in a study using simultaneous surface and intracranial recording found that electronegative sphenoidal spikes were recorded when electropositive hippocampal (HC) spikes were present. Although sphenoidal spikes are often associated with seizures originating from the hippocampus, they could also be present with seizures originating in the neocortical temporal or orbitofrontal area. Therefore, although sensitive, sphenoidal spikes are not very specific [41]. Spikes with maximum negativity over the mid/posterior temporal electrodes (T3/T4 or T5/T6) are more likely to originate from the temporal neocortex [22].

Sleep can increase the frequency of interictal spikes. Since IEDs are more prevalent in nonrapid eye movement (NREM) sleep than in wakefulness, Malow et al. reported that when combined with other investigations, IEDs recorded on overnight studies added prognostic data to the epilepsy surgery evaluation which was not provided by daytime EEGs $[101,102]$.

Previous studies have reported significant correlation of the lateralized temporal spikes to the side of the ictal onset [103]. These spikes are highly predictive of the ictal onset when they are associated with ipsilateral hippocampal atrophy. However, a unilateral scalp recorded IED may falsely lateralize the seizure origin; therefore, ictal recording is still necessary in presurgical evaluation [104, 105].

About one-third of patients with mTLE have bitemporal independent spikes or sharp waves mostly during nonREM sleep [96, 97]. Williamson et al. reviewed interictal scalp EEG abnormalities in mTLE and reported $42 \%$ of 67 patients to have bitemporal independent spikes or sharp wave [98]. Typical IED and/or intermittent slow waves in the anterior temporal region were present in $94 \%$ of patients. The strictly unilateral changes (observed in 52\%) correlated with the side of the seizure origin in $94 \%$ of patients. This was confirmed by subsequent depth EEG recording and surgical cure. The lateralized IEDs are often recorded during wakefulness and REM sleep. The more lateralized the spikes, the more predictive they are for the side of the seizure onset $[102,106]$.

Patients with bitemporal independent discharges could still be candidates for surgery and have a good surgical outcome when they have unilateral pathology, particularly when the side of the pathology and the ictal events are concordant. So et al. [107] reported the surgical outcome after a minimum of two years following temporal lobe surgery in 48 patients with bilateral independent temporal spikes. 14 were seizure free, 22 had greater than $50 \%$ seizure reduction, and three patients had less than three seizures a year. Chung et al. correlated the degree of lateralized IEDs with the surgical outcome after temporal lobectomy. When more than $90 \%$ of spikes were confined to one temporal lobe, $92 \%$ of patients had a good surgical outcome. When IED lateralization was less than $90 \%$, only half of patients achieved a good outcome [96].

\section{Ictal Scalp EEG in mTLE}

9.1. Seizure Onset. Gastaut [108] made an observation that "the attacks of psychomotor epilepsy are almost equally complex from an electroencephalographic and clinical point of view, and they cannot be reduced to a single mode of expression." Several factors such as the mesial versus lateral seizure onset, the underlying pathology, and the seizure propagation pattern could account for the variable EEG expression [23].

Some studies have indicated that the lateralized IED to the side of the temporal lobe lesion on MRI could be sufficient for surgical resection [104, 109]. However, in majority 




FIgure 1: Temporal Intermittent Rhythmic Delta Activity (TIRDA) recorded in a patient with left temporal lobe epilepsy. This patient also had epileptiform discharges in the left anterior temporal region. ( $\mathrm{HFF}=15 \mathrm{~Hz}, \mathrm{LFF}=0.5 \mathrm{~Hz}$, and sensitivity $=7 \mu \mathrm{V} / \mathrm{mm}$ ).

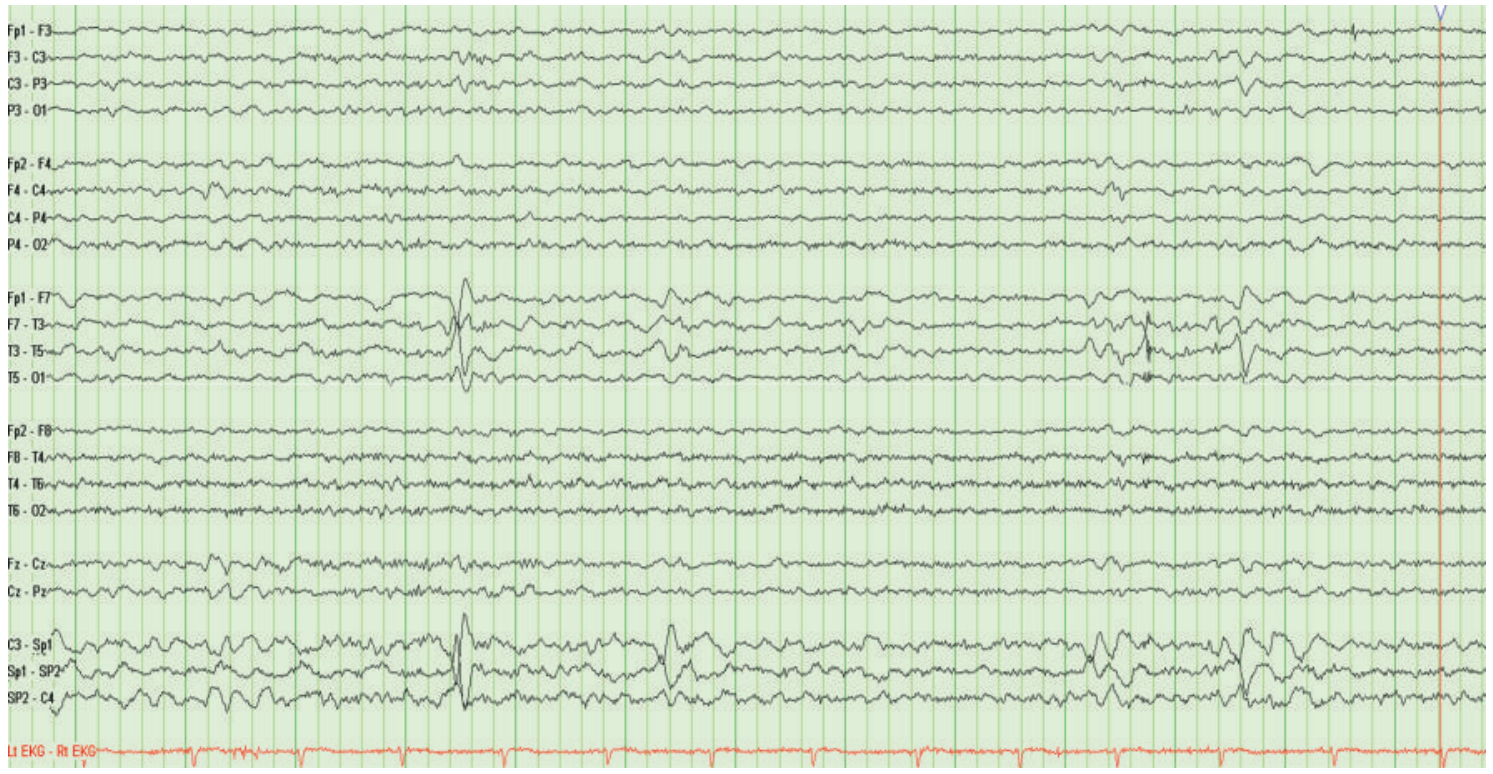

FIGURE 2: A 29-year-old woman with history of complex partial seizures. Scalp EEG demonstrates sharp waves phase reversing over F7-T3 and Sphenoidal SP1 electrodes. $(\mathrm{HFF}=15, \mathrm{LFF}=0.5$, and sensitivity $=10 \mu \mathrm{V} / \mathrm{mm})$.

of patients with negative MRI, nonlateralizing IED or bilateral epileptiform discharges, ictal EEG recording provides the most important data regarding the seizure onset in presurgical evaluation of patients with refractory temporal lobe epilepsy.

EEG is often unremarkable during aura or at the beginning of the abnormal clinical behaviour [98]. The ictal EEG manifestation of limbic seizures could have a nonspecific beginning of low-voltage fast (electrodecrement) activity, with focal or regional background attenuation. The lowvoltage fast activity is seen in up to $25 \%$ of patients and does not provide lateralizing/localizing value $[109,110]$.

Blume et al. studied the EEG morphology of focal seizures. He found that other than initial attenuation, there were sinusoidal waveforms in $47 \%$ and repetitive epileptiform discharges in up to $39 \%$ of patients. Both EEG patterns were observed in $15 \%$ of patients. In the last stage of the seizure, the frequency decreased, while the amplitude had 
often increased [111]. Not all patients in this study had temporal lobe onset seizures.

In seizures recorded by subdural electrodes, Blume and Kaibara [112] reported "start-stop-start" pattern at the seizure onset which was also reported in $13 \%$ of patients recorded by scalp electrodes in another study [113]. If the initial part of the seizure onset is missed, the restart of the EEG changes could be incorrectly considered as the seizure onset.

Williamson et al. found buildup of lateralized rhythmic $5-10 \mathrm{~Hz}$ sharp activity in $81 \%$ of seizures in mTLE. This appeared within 30 seconds of the first objective or subjective clinical symptoms and signs of a seizure and was observed in the anterior-inferior temporal scalp electrodes. This pattern was concordant with the side of the seizure onset in 47 out of 67 patients, and it was discordant in five patients. This characteristic rhythm occurs in $82-94 \%$ of patients with mTLE and has $95 \%$ specificity for lateralizing the seizure onset (Figure 3) [98, 114-116].

The frequency of the ictal onset EEG in neocortical temporal lobe seizures is often in the $2-5 \mathrm{~Hz}$ range [117]. The temporal-sphenoidal pattern is less common. There is also less stability of frequency and voltage. Absence of the ictal scalp discharge is more common in nTLE than in mTLE $[114,118,119]$. The overlap in EEG findings between mTLE and nTLE in limbic seizures does not allow confident distinction between the two by ictal EEG alone [55].

\section{Reliability of Interictal and Ictal Scalp EEG Findings for Surgical Decision}

10.1. Accuracy of IEDs. Recent literature agrees that IEDs are valuable predictors of the site of the seizure onset. Previous studies relied mainly on scalp EEG abnormalities or clinical picture to recognize temporal lobe seizures $[120,121]$. Several recent studies have reported a strong association between the location and frequency of IEDs and the side of the seizure onset $[91,96,103]$. One study reported $93 \%$ of 56 patients with seizures originating from one temporal lobe had IED exclusively or predominantly in that temporal lobe [112].

Casino et al. studied 159 patients with intractable TLE who underwent anterior temporal lobectomy between 1988 and 1993. The epileptogenic temporal lobe was determined by ictal recording on long-term monitoring. Routine EEG revealed temporal lobe epileptiform discharges in 123 patients which correlated with the temporal lobe of seizure origin $(P<0.0001)$ and the results of MRI volumetric studies $(P<0.0001)$. However, it was discordant with interictal findings in another 20 patients. They concluded that in patients with TLE, IEDs localized to one temporal lobe on serial routine EEGs or during long-term recording may be adequate to identify the epileptogenic zone [109].

Patients with unifocal or highly predominant interictal temporal spike or sharp wave are very likely to have focal ictal onset from the same temporal lobe. However, some studies have reported $20-40 \%$ false lateralization in those with predominant lateralized interictal finding on scalp EEG. Hence, the unilateral surface temporal IED did not exclude the possibility of bilateral independent ictal onset as documented by subsequent intracranial recordings [122].

The overall accuracy of scalp lateralization has been reported to be $60-83 \%$. Barba et al. analyzed the differentiating features between temporal lobe (TL) and temporal plus $(\mathrm{T}+)$ epilepsy and concluded that interictal spikes could not definitely differentiate the TL from T+ group [123]. However in $\mathrm{T}+$ group, scalp EEG recordings demonstrated more precentral interictal abnormalities and were topographically different from the frontotemporal sharp waves described in mTLE. In T+ group, there were more frequent bilateral interictal abnormalities previously reported to be associated with worse surgical outcome [124]. Temporal lobe interictal spikes or ictal EEG onset did not definitively rule out the possibility of $\mathrm{T}+$ epilepsies similar to the study reported by Aghakhani et al. [125]. Interictal and ictal scalp data guide towards intracranial recording in order to delineate the epileptogenic zone for resection.

The presence of temporally located interictal abnormality in patients with extratemporal lobe epilepsy has been discussed in several studies [126-129].

10.2. Ictal Accuracy in Scalp EEG. Risinger et al. retrospectively analysed 706 noninvasive ictal recordings from 110 patients to study the reliability of scalp/sphenoidal ictal recording for localization. These patients subsequently underwent depth electrode study. The authors found that $52 \%$ of patients displayed $5 \mathrm{~Hz}$ or faster frequency maximum over on sphenoidal or temporal electrode early at the seizure onset. This pattern was predictive of the side of the ipsilateral temporal lobe seizure onset identified by depth recording in $82 \%$ of these patients. When exclusively focal on scalp, the predictive value of this pattern was $94 \%$. This number reduced to $67 \%$ when mixed focal and nonfocal patterns were present. The interobserver reliability among the three interpreters was excellent. False lateralization of the $5 \mathrm{~Hz}$ frequency was seen in $18 \%$ of patients. The authors concluded that this pattern was misleading in minority of patients; however, it cannot be used in isolation for seizure localization [114].

Interobserver reliability in localizing ictal scalp EEGs comparing to the ictal data from depth recording was also studied by Spencer et al. [130]. They reviewed 144 seizures in 54 patients recorded by combination of scalp and intracranial electrodes and interpreted by three blinded electroencephalographers. The Kappa statistics, a measurement of index of agreement, found the reliability of lateralization between pairs of EEG interpreters was "fair to good." However, this measure was "poor to fair" between interpreters when the lobe of seizure onset was considered.

Walczak et al. [116] used the seizure outcome as "gold standard" for the accuracy of the ictal EEG in identifying the epileptogenic zone. In retrospective analysis of 137 scalp ictal EEGs from 35 patients with temporal lobe resection, three blinded electroencephalographers correctly identified the side of the seizure onset in $76-83 \%$ of the 119 temporal lobe seizures with Kappa statistics of "fair to good." In analysis 


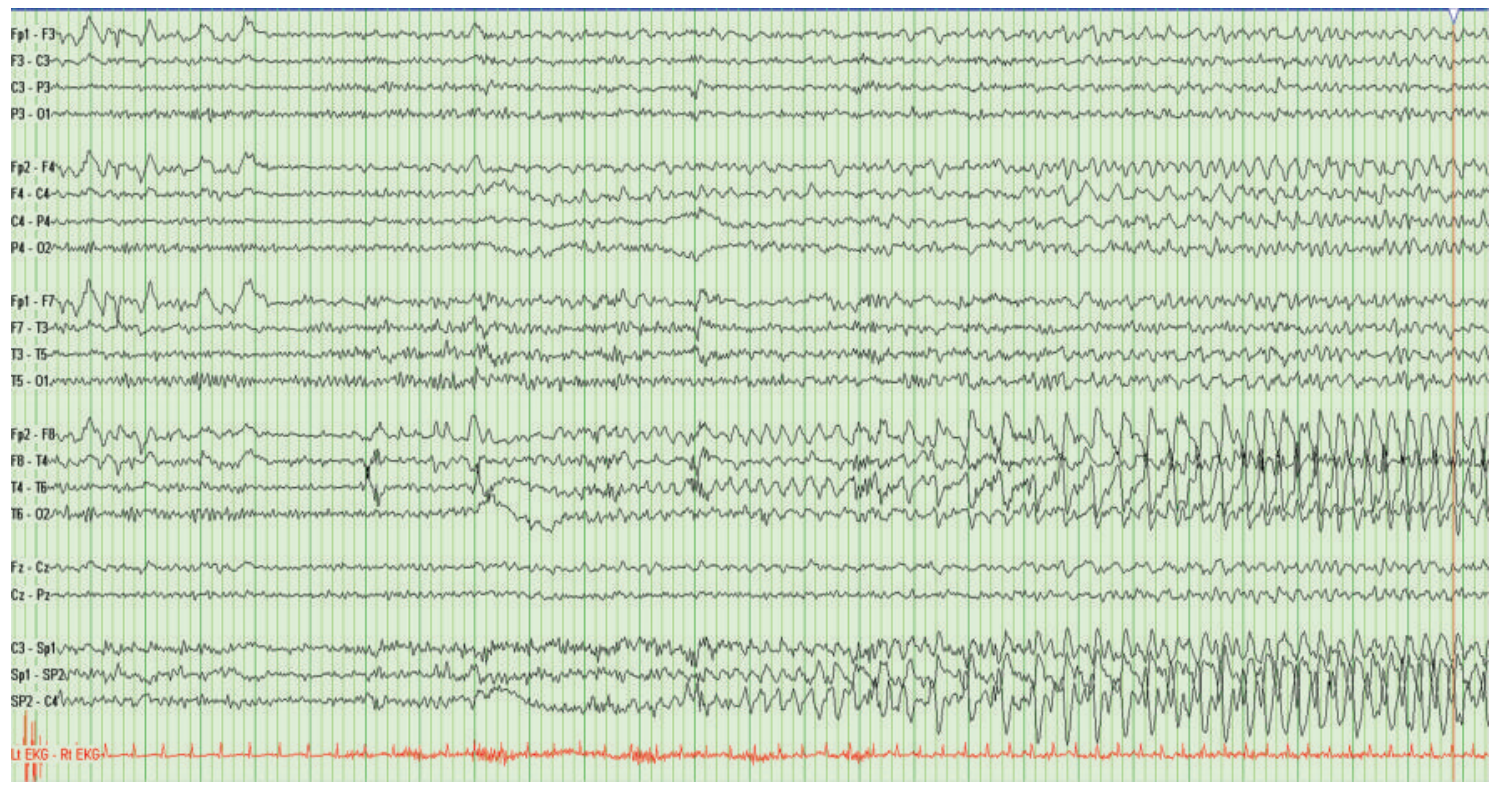

FIGURE 3: A 32-year-old man with right mesial temporal lobe epilepsy. EEG demonstrates generalized and predominantly right-sided electrodecrement followed by buildup of rhythmic activity in the right temporal region. He had a successful surgical result and has been seizure free $(\mathrm{HFF}=70, \mathrm{LFF}=1$, and sensitivity $=10 \mu \mathrm{V} / \mathrm{mm})$.

restricted to only seizures that had lateralizing features, the accuracy of lateralization was $93-98 \%$. Among individual EEG patterns, alpha theta frequency at the seizure onset was most accurate.

Differentiating mTLE from nTLE by clinical and scalp EEG characteristic could be quite challenging. However, in the latter the EEG changes are more widely distributed, with more involvement of the frontocentral electrodes and more variable frequency and amplitude at the onset [98].

\section{Seizure Detection by Mathematical Models}

Digital EEG technology has been pivotal in improving the detection of spikes and seizures mainly during continuous video/EEG monitoring. Gotman has been one of the pioneers in advancement of seizure detection technology and its progressive improvement and state-dependent algorithms [131]. Spatiotemporal digital filtering has allowed elimination of noncerebral artifacts analysis of the EEG signal at seizure onset improving the detection and lateralization $[132,133]$. One has to be careful not to eliminate rhythmic cerebral slow waves when using EEG filters.

There has been much information regarding the localization and the frequency of the seizure onset and propagation using the computerized methodical models such as wavelet transforms [134]. Using a novel wavelet-based algorithm (wavelet packet transform) for real-time detection of epileptic seizures on scalp EEG, we were able to improve the seizure detection sensitivity to $90.5 \%$, a false detection rate of $0.51 \mathrm{~h}$ 1 and a median detection delay of 7 seconds. This algorithm could also lateralize the seizure focus in patients with seizures originating from the temporal lobes [135].

\section{Seizure Propagation}

Lieb et al. studied the pattern of spread in 24 patients with mesial temporal lobe seizures who underwent depth electrode study. The authors reported that the seizure propagation and the EEG expression can be influenced by the state of the patient (awake or sleep), the use of anti-seizure drugs and unilateral versus multifocal epilepsy [136]. Medial versus lateral location of the seizure onset and the type of pathology (e.g., HS or neoplasm) also determine the pattern of seizure propagation on scalp EEG in TLE.

The localization of the epileptogenic zone and the spread pattern by simultaneous scalp and intracranial recordings was reported by Sakai et al. [137]. In this study, they recorded the ictal and the spread pattern in 28 medial and 14 lateral temporal onset seizures in 30 patients with mTLE and 8 patients with nTLE. They concluded that ictal discharges originating in medial temporal lobes propagated to the lateral part on the same side and the lateral part of the opposite side via contralateral medial area. Although this gives a reasonable accuracy in predicting the side of the seizure focus, at times the latency of spread to the ipsilateral temporal region is the same or even longer than contralateral spread. This results in observing earlier discharges on the contralateral side. Therefore, the side showing the leading ictal activity may not necessarily be the side of the seizure origin.

\section{Postictal EEG Changes in mTLE}

The ictal onset on scalp recorded EEG can be obscured by variety of artifacts. Therefore, sometimes postictal changes 
could provide important information for lateralization of the seizure onset. Postictal EEG changes in TLE could be in the form of unilateral or bilateral slowing. Lateralized postictal slow waves could be present in up to $70 \%$ of patients with mTLE. When present, it has a lateralizing value of about $90 \%$. Williamson et al. found lateralized postictal slowing in 45 of 67 patients (67\%), and it was always concordant with the side of the seizure origin [98].

Kaibara and Blume [89] in a study of patients with TLE reported postictal changes in 69\%. These changes consisted of regional delta activity and regional attenuation or activation of spikes. They were ipsilateral to the side of the seizure onset in all cases. Jan et al. analysed the relationship of the postictal delta activity to the side of the seizure onset in patients with TLE. The two EEG interpreters were blinded to the clinical and EEG data. Lateralized postictal delta was observed in $64 \%$ of all ictal EEGs and $76 \%$ in at least one record from 22 patients. There was a strong interobserver agreement. When present, the postictal delta activity was concordant with the side of surgery in $96 \%$ of the EEGs and strongly predicted the seizure onset [138].

\section{Intracranial Recordings in mTLE}

14.1. Indications. In most cases of medial temporal lobe epilepsy standard scalp EEG with the use of sphenoidal electrodes and the MRI evidence of HS will be sufficient to identify the seizure focus prior to surgery. However, considering the limitation of the scalp EEG, that is, contamination with muscle and movement artefact, equivocal scalp EEG changes, interobserver disagreement, and the possibility of false lateralization, the recordings could be obscured or misleading. The EEG recording with placement of the intracranial electrodes was introduced in 1960s and 1970s. King and Spencer described invasive EEG recording in mTLE [139].

Risinger et al. reported that only $19 \%$ of auras and 10\% of subclinical seizures could have surface EEG expression. Using both surface and depth electrodes, they found that a large number of seizures revealed no scalp EEG expression at the onset when it already appeared on depth electrodes, and scalp EEG showed bilateral and nonlateralizing changes [114].

The advantages and disadvantages of this technique were recently reviewed in the study by Dubeau and MacLachlan [140]. Table 5 summarises the indications for intracranial recordings.

Intracranial recording is artefact free and more specific and has very good temporal resolution [141, 142]. It increases the yield of detecting IEDs when scalp EEG fails. It also allows identification of the seizure focus with more accuracy and reduces the risk of false lateralization. This is particularly important in those patients with ambiguous seizure onset, and with rapid seizure transfer from one to the other temporal lobe. In order to obtain accurate electrophysiological information, a working hypothesis must be developed before proceeding to invasive intracranial recording. Figure 4 demonstrates an example of seizure recorded by subdural electrodes.
TABLE 5: Indications for intracranial EEG recordings [140].

Temporal lobe syndrome with bilateral independent interictal or ictal abnormalities

Discordant data seizures originating from the side contralateral to the MRI abnormality or interictal discharges

Seizures with undetermined side of onset

Mesial versus neocortical seizure onset

Mesial versus onset in the neighbouring structures "temporal plus epilepsies"

Occipitotemporal epilepsy

To avoid false lateralization

In cases of mTLE with severe HS, the EEG changes of the seizure onset may not propagate to the ipsilateral scalp and instead, some seconds later the ictal discharge appears on the contralateral side resulting in false lateralization. False EEG lateralization in patients with temporal lobe seizures was reported as high as $10 \%$ [143]. Intracranial recording is crucial in such patients.

Temporal plus $(\mathrm{T}+)$ epilepsies has recently been addressed and reviewed by Munari et al. [144], Kahane et al. [15], and Ryvlin and Kahane [14]. In one study of clinical manifestations of insular lobe seizures, temporal lobe surgery alone was unsuccessful in controlling seizures that originated from the insula [145]. Barba et al. [123] retrospectively analysed clinical and EEG findings in 80 consecutive patients who were thought to suffer from nonlesional temporal lobe seizures. Stereotactic intracerebral EEG (SEEG) recordings could identify two groups of "purely temporal" (TL group) and temporal plus ( $\mathrm{T}+$ group). They concluded that the general clinical features or MRI data including the presence of HS could not differentiate the two groups. However, both ictal clinical symptoms and scalp EEG findings significantly differentiated TL from T+ patients.

When interictal and ictal EEG were correlated with MRI lesions in focal epilepsy, Remi et al. found the localization of IEDs was most congruent with MRI lesions in the temporal lobe compared to the lesions in other lobes. Temporal lobe IEDs are also frequently present in extratemporal lobe epilepsies; therefore, temporal IEDs could be misleading [146].

14.2. The Types of Intracranial Electrodes. They include depth, subdural, epidural, and foramen ovale electrodes. Depth electrodes are inserted through a bur hole and can be removed at the bedside with caution. They penetrate the brain and lie within the cortex. They are utilized for recording from buried cortex such as amygdala and hippocampus and sometimes orbitofrontal cortex. Foramen ovale electrodes are similar to the depth electrodes and are inserted under fluoroscopy in radiology or in the operating room.

Subdural electrodes lie on the pial surface of the brain. The strips have a single or double row of contacts. They can be placed through a bur hole and lie directly to the proximity of the brain. They record from gyral surface over multiple areas and from the epidural or subdural spaces. Subdural 




FIGURE 4: A seizure recorded using subdural electrodes. The top 24 channels are from the gird and subtemporal electrodes on the right. The lower 24 channels are from the left side. The lowest six channels are from the left subtemporal contacts. EEG demonstrates high-amplitude ictal discharges over the left anterior contacts of the middle subtemporal electrode (hippocampus) followed by high-frequency discharges over all eight contacts of the two subtemporal and the two contacts of the most anterior subtemporal electrode $($ amygdala $)(\mathrm{HFF}=70, \mathrm{LFF}=$ 1 , and sensitivity $=150 \mu \mathrm{V} / \mathrm{mm}$ ).

grids are larger in size and monitor a larger area of cortex. They are also used for brain mapping. Subdural strips are better tolerated than subdural grids and can be removed at the bed side; however, grids have to be removed in the operating room.

The chronic intracranial electrodes have $1-4 \%$ risk of morbidity and mortality. This includes haemorrhage and infectio; brain swelling and herniation [55].

14.3. Intracranial EEG Features in $m$ TLE. Pacia and Ebersole [147] in a comprehensive study of combined and simultaneous scalp-intracranial study using depth, subdural strips and grids, analysed the intracranial EEG features responsible for producing the three different scalp EEG manifestations (types 1, 2, and 3). They observed that seizures starting at the hippocampal contacts of the depth electrode, showed ictal rhythms recorded from the mesial contacts of the subdural strip electrodes. Simultaneous scalp recording showed no representation or diffuse disruption of the background.

The 6-7 Hz rhythm only appeared in the inferior and basal scalp electrodes when the seizure spread to the basal and inferolateral subdural contacts. The authors concluded that $5-9 \mathrm{~Hz}$ frequency recorded on the scalp (type 1a) electrodes was the result of the synchronous recruitment of the adjacent inferolateral temporal neocortex, and it was highly associated with seizure onset in the hippocampus, but it was not a direct manifestation of the hippocampal ictal activity. Seizures beginning in the temporal neocortical region did not have this manifestation until the hippocampus was involved.

Type 2 pattern had slower $(<5 \mathrm{~Hz})$ frequency and mostly generated in the neocortical structures. There was an initial focal or regional low-voltage $20-40 \mathrm{~Hz}$ rhythm and widespread background flattening on interictal electrodes. On the scalp, only attenuation of the normal rhythm was observed, and it was followed by a slow and irregular rhythm. By intracranial EEG this type showed an extrahippocampal and probably temporal neocortical ictal origin. Interestingly, three of the patients with mesial temporal onset seizures showed similar scalp EEG manifestations.

The type 3 scalp EEG pattern, characterized by diffuse slowing or attenuation of the background, without typical rhythmic patterns resulted from seizure activity confined to the hippocampus in four of the seven and with the neocortical seizure onset in the other three patients. This scalp seizure onset pattern was seen when there was rapid seizure propagation to the contralateral temporal lobe or when cortical activity failed to achieve widespread synchrony.

Location of the seizure onset is also related to the degree of pathology in the medial temporal region. In a depth electrode study, the ictal discharges were restricted to the hippocampus in severe HS [148]. In other medial temporal pathologies such as tumours, there are often more than one ictal onset zone usually detected in the tissue adjacent to the tumor rather than within the tumor or in the mesial temporal structures; therefore, the accurate localization of 
the ictal onset is critical for complete surgical resection of the epileptogenic zone and better surgical outcome [149].

\section{Magnetoencephalography (MEG)}

MEG has been used for localization of the epileptic spikes. EEG records the extracellular electrical current which is subject to distortion by different intervening tissues. MEG detects magnetic fields generated by neurons in the walls of cortical sulci. These neurons produce current dipoles tangential to the skull. However, currents produced by neurons at the tip of the gyri that are radial to the skull do not influence MEG. The intervening tissues can distort the electrical currents produced in the sulci and gyri when recorded by EEG. However, MEG can easily record magnetic fields without distortion by different tissues such as bone, muscle, and skin. In addition, MEG measurements are absolute without active reference contamination compared to differential measurement by EEG. Several studies have reported the benefits of MEG in clinical epilepsy and TLE $[150,151]$.

MEG could help to reduce the need for invasive monitoring in focal epilepsy. It has been concordant with localization by intracranial electrodes [152]. MEG has also been most helpful in non-lesional extratemporal lobe epilepsy [153]. Ebersole et al. found MEG spikes in the sublobar temporal and frontal regions in 30 patients. Half of these patients did not have focal abnormality such as hippocampal atrophy in their MRI/CT studies [154].

MEG dipole modeling identified two patient groups in mesial temporal lobe epilepsy. In one group spikes were modeled by anterior temporal vertical dipoles indicating epileptic activity in the medial basal temporal area and in another group spikes modeled by anterior temporal horizontal dipoles indicating the epileptic activity in the temporal pole and lateral temporal area. The first group had more consistent localization in the ipsilateral temporal lobe; however, half of the other group had bitemporal spikes on CV-EEG monitoring [155].

MEG is another noninvasive technology that provides simple method of recording the brain activity with spatial and temporal resolution of spikes superior but complimentary to traditional EEG. However considering the high cost of MEG, EEG is still an inexpensive and easily available technology to monitor the activity of the brain.

\section{Conclusion}

EEG has been pivotal in the diagnosis, classification, and management of epilepsy since the early part of the last century. It has a special role in the presurgical evaluation of patients with focal epilepsy refractory to medications. Serial EEG is sensitive and specific for the diagnosis of epilepsy. In patients who withdraw medications after a period of seizure freedom following surgery, the presence of IEDs on EEG could be predictive of seizure recurrence.

In mTLE, scalp EEG in most instances can lateralize and localize the seizure focus. Other than epileptiform discharges, abnormal lateralized EEG patterns such as TIRDA could be quite specific for the ipsilateral seizure onset. Interictal spikes or sharp waves are often unilateral; however, in about the third of patients they are bitemporal independent.

Ictal EEG patterns appear in the form of background attenuation, start-stop phenomena, $5-10 \mathrm{~Hz}$ rhythmic sinusoidal waveforms or repetitive sharp waves or spikes. The $2-5 \mathrm{~Hz}$ lateralized activity is often due to lateral neocortical focus. Postictal slowing if lateralized, is often ipsilateral to the seizure focus.

Invasive recording with intracranial electrode placement is required when the information from the surface recording is insufficient or nonconcordant for detection of the epileptogenic zone. The indications were highlighted in this paper. Implanted subdural strips, grids, or depth electrodes can provide information from medial or lateral temporal electrodes and could also help identifying temporal plus epilepsies with clinical semiology similar to mTLE.

The combination of clinical and MRI information with the EEG findings are most useful in identifying the seizure focus for surgical treatment of mesial temporal lobe epilepsy.

\section{References}

[1] J. Roger, F. E. Dreifuss, M. Martinez-Lage et al., "Proposal for revised classification of epilepsies and epileptic syndromes. Commission on classification and terminology of the International League Against Epilepsy," Epilepsia, vol. 30, no. 4, pp. 389-399, 1989.

[2] H. G. Wieser, "Mesial temporal lobe epilepsy with hippocampal sclerosis," Epilepsia, vol. 45, no. 6, pp. 695-714, 2004.

[3] A. T. Berg, S. F. Berkovic, M. J. Brodie et al., "Revised terminology and concepts for organization of seizures and epilepsies: report of the ilae commission on classification and terminology, 2005-2009," Epilepsia, vol. 51, no. 4, pp. 676$685,2010$.

[4] T. L. Babb and W. J. Brown, "Pathological findings in epilepsy," in Surgical Treatment of Epilepsies, J. Engel Jr., Ed., pp. 511-540, Raven, New York, NY, USA, 1987.

[5] J. Engel Jr., P. D. Williamson, and H. G. Weiser, "Mesial temporal lobe epilepsy with hippocampal sclerosis," in Epilepsy: A Comprehensive Textbook, J. Engel Jr. and T. A. Pedley, Eds., pp. 2487-2493, Lippincott William and Wilkins, Philadelphia, Pa, USA, 2nd edition, 2008.

[6] G. D. Jackson, S. F. Berkovic, B. M. Tress, R. M. Kalnins, G. C. A. Fabinyi, and P. F. Bladin, "Hippocampal sclerosis can be reliably detected by magnetic resonance imaging," Neurology, vol. 40, no. 12, pp. 1869-1875, 1990.

[7] R. I. Kuzniecky and G. D. Jackson, Magnetic Resonance in Epilepsy. Neuroimaging Techniques, Elsevier, London, UK, 2nd edition, 2005.

[8] F. Semah, M. C. Picot, C. Adam et al., "Is the underlying cause of epilepsy a major prognostic factor for recurrence?" Neurology, vol. 51, no. 5, pp. 1256-1262, 1998.

[9] L. J. Stephen, P. Kwan, and M. J. Brodie, "Does the cause of localisation-related epilepsy influence the response to antiepileptic drug treatment?" Epilepsia, vol. 42, no. 3, pp. 357-362, 2001.

[10] S. F. Berkovic, R. A. Howell, and J. L. Hopper, "Familial temporal lobe epilepsy: a new syndrome with adolescent/adult onset and a benign course," in Epileptic Seizures and Syndromes, P. Wolf, Ed., pp. 257-263, John Libbey, London, UK, 1994. 
[11] S. F. Berkovic, A. McIntosh, R. Anne Howell, A. Mitchell, L. J. Sheffield, and J. L. Hopper, "Familial temporal lobe epilepsy: a common disorder identified in twins," Annals of Neurology, vol. 40, no. 2, pp. 227-235, 1996.

[12] E. Kobayashi, M. D. D’Agostino, I. Lopes-Cendes et al., "Outcome of surgical treatment in familial mesial temporal lobe epilepsy," Epilepsia, vol. 44, no. 8, pp. 1080-1084, 2003.

[13] J. A. French, P. D. Williamson, V. M. Thadani et al., "Characteristics of medial temporal lobe epilepsy: I. Results of history and physical examination," Annals of Neurology, vol. 34, no. 6, pp. 774-780, 1993.

[14] P. Ryvlin and P. Kahane, "The hidden causes of surgeryresistant temporal lobe epilepsy: extratemporal or temporal plus?" Current Opinion in Neurology, vol. 18, no. 2, pp. 125127, 2005.

[15] P. Kahane, J. C. Huot, D. Hoffmann, G. L. Russo, A. L. Benabid, and C. Munari, "Perisylvian cortex involvement in seizures affecting thetemporal lobe," in Limbic Seizure in Children, G. Avanzini, A. Beumonoir, and L. Mira, Eds., pp. 115-127, John Libbey, 2001.

[16] S. Wiebe, W. T. Blume, J. P. Girvin, and M. Eliasziw, "A randomized, controlled trial of surgery for temporal-lobe epilepsy," The New England Journal of Medicine, vol. 345, no. 5, pp. 311-318, 2001.

[17] J. Engel Jr., S. Wiebe, J. French et al., "Practice parameter: temporal lobe and localized neocortical resections for epilepsy-report of the quality standards subcommittee of the american academy of neurology, in association with the american epilepsy society and the american association of neurological surgeons," Neurology, vol. 60, no. 4, pp. 538$547,2003$.

[18] J. F. Téllez-Zenteno, R. Dhar, and S. Wiebe, "Long-term seizure outcomes following epilepsy surgery: a systematic review and meta-analysis," Brain, vol. 128, no. 5, pp. 1188 1198, 2005.

[19] J. de Tisi, G. S. Bell, J. L. Peacock et al., "The long-term outcome of adult epilepsy surgery, patterns of seizure remission, and relapse: a cohort study," The Lancet, vol. 378, no. 9800, pp. 1388-1395, 2011.

[20] S. Mittal, J. L. Montes, J. P. Farmer et al., "Long-term outcome after surgical treatment of temporal lobe epilepsy in children," Journal of Neurosurgery, vol. 103, no. 5, supplement, pp. 401-412, 2005.

[21] J. T. Lerner, N. Salamon, J. S. Hauptman et al., "Assessment and surgical outcomes for mild type I and severe type II cortical dysplasia: a critical review and the UCLA experience," Epilepsia, vol. 50, no. 6, pp. 1310-1335, 2009.

[22] M. Sadler and R. Desbiens, "Scalp EEG in temporal lobe epilepsy surgery," Canadian Journal of Neurological Sciences, vol. 27, supplement, pp. S22-S28, 2000.

[23] M. Jan, M. Sadler, and S. Rahey, "Electroencephalographic features of temporal lobe epilepsy," Canadian Journal of Neurological Sciences, vol. 37, no. 4, pp. 439-448, 2010.

[24] J. Gotman, E. Kobayashi, A. P. Bagshaw, C. G. Bénar, and F. Dubeau, "Combining EEG and FMRI: a multimodal tool for epilepsy research," Journal of Magnetic Resonance Imaging, vol. 23, no. 6, pp. 906-920, 2006.

[25] G. D. Jackson, R. S. Breillmann, and R. I. Kuznieky, “Temporal lobe epilepsy," in Magnetic Resonance in Epilepsy. Neuroimaging Techniques, R. I. Kuznieky and G. D. Jackson, Eds., pp. 99-176, Elsevier, London, UK, 2nd edition, 2005.

[26] N. D. Chiaravalloti and G. Glosser, "Material-specific memory changes after anterior temporal lobectomy as predicted by the intracarotid amobarbital test," Epilepsia, vol. 42, no. 7, pp. 902-911, 2001.

[27] T. A. Tran, S. S. Spencer, M. Javidan, S. Pacia, D. Marks, and D. D. Spencer, "Significance of spikes recorded on intraoperative electrocorticography in patients with brain tumor and epilepsy," Epilepsia, vol. 38, no. 10, pp. 1132-1139, 1997.

[28] F. A. Gibbs, H. Davis, and W. G. Lennox, "The electroencephalogram in epilepsy and in conditions of impaired consciousness," Archives of Neurology and Psychiatry, vol. 34, no. 6, pp. 1133-1148, 1935.

[29] J. Lewis, Something Hidden: A Biography of Wilder Penfield, Doubleday, Garden City, NY, USA, 1981.

[30] W. A. Kennedy and D. Hill, "The surgical prognostic significance of the electroencephalographic prediction of Ammon's horn sclerosis in epileptics," Journal of Neurology, Neurosurgery, and Psychiatry, vol. 21, no. 1, pp. 24-30, 1958.

[31] P. Bailey and F. A. Gibbs, "The surgical treatment of psychomotor epilepsy," Journal of the American Medical Association, vol. 145, no. 6, pp. 365-370, 1951.

[32] J. Engel, M. V. Driver, and M. A. Falconer, "Electrophysiological correlates of pathology and surgical results in temporal lobe epilepsy," Brain, vol. 98, no. 1, pp. 129-156, 1975.

[33] D. S. Gorney, “The practical guide to digital EEG," American Journal of EEG Technology, vol. 32, no. 4, pp. 260-289, 1992.

[34] J. Gotman, "Automatic recognition of interictal spikes," in Long Term Monitoring in Epilepsy, J. Gotman, J. R. Ives, and P. Gloor, Eds., pp. 93-114, Elsevier, Amsterdam, The Netherlands, 1958.

[35] J. Gotman, "Seizure recognition and analysis," in Long Term Monitoring in Epilepsy, J. Gotman, J. R. Ives, and P. Gloor, Eds., pp. 133-145, Elsevier, Amsterdam, The Netherlands, 1958.

[36] H. Jasper, "The ten-twenty electrode system of the International Federation," Electroencephalography and Clinical Neurophysiology, vol. 10, no. 2, pp. 371-375, 1958.

[37] R. M. Sadler and J. Goodwin, "Multiple electrodes for detecting spikes in partial complex seizures," Canadian Journal of Neurological Sciences, vol. 16, no. 3, pp. 326-329, 1989.

[38] H. H. Morris III, H. Luders, and R. P. Lesser, "The value of closely spaced scalp electrodes in the localization of epileptiform foci: a study of 26 patients with complex partial seizures," Electroencephalography and Clinical Neurophysiology, vol. 63, no. 2, pp. 107-111, 1987.

[39] A. M. Kanner, L. Ramirez, and J. C. Jones, "The utility of placing sphenoidal electrodes under the foramen ovale with fluoroscopic guidance," Journal of Clinical Neurophysiology, vol. 12, no. 1, pp. 72-81, 1995.

[40] C. D. Binnie, D. Marston, C. E. Polkey, and D. Amin, "Distribution of temporal spikes in relation to the sphenoidal electrode," Electroencephalography and Clinical Neurophysiology, vol. 73, no. 5, pp. 403-409, 1989.

[41] R. J. Wilkus, P. M. Thompson, and D. G. Vossler, "Quantitative comparison of ictal EEG from sphenoidal, minisphenoidal, anterior temporal, and midtemporal electrodes," Epilepsia, vol. 32, supplement 13, pp. 100-101, 1991.

[42] J. R. Ives, F. W. Drislane, S. C. Schachter et al., "Comparison of coronal sphenoidal versus standard anteroposterior temporal montage in the EEG recording of temporal lobe seizures," Electroencephalography and Clinical Neurophysiology, vol. 98, no. 5, pp. 417-421, 1996.

[43] D. A. Marks, A. Katz, J. Booke, D. D. Spencer, and S. S. Spencer, "Comparison and correlation of surface and 
sphenoidal electrodes with simultaneous intracranial recording: an interictal study," Electroencephalography and Clinical Neurophysiology, vol. 82, no. 1, pp. 23-29, 1992.

[44] C. E. Miley and F. M. Forster, "Activation of partial complex seizures by hyperventilation," Archives of Neurology, vol. 34, no. 6, pp. 371-373, 1977.

[45] K. L. Pratt, R. H. Mattson, N. J. Weikers, and R. Williams, "EEG activation of epileptics following sleep deprivation: a prospective study of 114 cases," Electroencephalography and Clinical Neurophysiology, vol. 24, no. 1, pp. 11-15, 1968.

[46] J. Rowan, R. J. Veldhuisen, and N. J. D. Nagelkerke, "Comparative evolution of sleep deprivation and sedated sleep EEGs as diagnostic aids in epilepsy," Electroencephalography and Clinical Neurophysiology, vol. 54, pp. 357-364, 1982.

[47] B. Santoshkumar, J. J. Chong, W. T. Blume et al., "Prevalence of benign epileptiform variants," Clinical Neurophysiology, vol. 120, no. 5, pp. 856-861, 2009.

[48] J. C. White, J. W. Langston, and T. A. Pedley, "Benign epileptiform transients of sleep. clarification of the small sharp spike controversy," Neurology, vol. 27, no. 11, pp. 1061-1068, 1977.

[49] J. Reiher, M. Lebel, D. W. Klass et al., "Small sharp spikes (SSS): reassessment of electroencephalographic characteristics and clinical significance," Electroencephalography and Clinical Neurophysiology, vol. 43, p. 775, 1977.

[50] J. Reiher and M. Lebel, "Wicket spikes: clinical correlates of a previously undescribed EEG pattern," Canadian Journal of Neurological Sciences, vol. 4, no. 1, pp. 39-47, 1977.

[51] I. J. Lipman and J. R. Hughes, "Rhythmic mid-temporal discharges. an electro-clinical study," Electroencephalography and Clinical Neurophysiology, vol. 27, no. 1, pp. 43-47, 1969.

[52] J. W. Kozelka and T. A. Pedley, "Beta and Mu rhythms," Journal of Clinical Neurophysiology, vol. 7, no. 2, pp. 191-207, 1990.

[53] C. R. Miller, B. F. Westmoreland, and D. W. Klass, "Subclinical rhythmic EEG discharge of adults (SREDA): further observations," American Journal of EEG Technology, vol. 25, no. 4, pp. 217-224, 1985.

[54] B. F. Westmoreland and D. W. Klass, "Unusual variants of subclinical rhythmic electrographic discharge of adults (SREDA)," Electroencephalography and Clinical Neurophysiology, vol. 102, no. 1, pp. 1-4, 1997.

[55] T. A. Pedley, A. Mendiratta, and T. S. Walczak, "Seizures and epilepsy," in Current Practice of Clinical Electroencephalography, J. S. Ebersole and T. A. Pedley, Eds., pp. 506-587, Lipincott Williams and Wilkins, Philadelphia, Pa, USA, 3rd edition, 2003.

[56] M. Sundaram, R. M. Sadler, G. B. Young, and N. Pillay, "EEG in epilepsy: current perspectives," Canadian Journal of Neurological Sciences, vol. 26, no. 4, pp. 255-262, 1999.

[57] H. Meierkord, B. Will, D. Fish, and S. Shorvon, "The clinical features and prognosis of pseudoseizures diagnosed using video-EEG telemetry," Neurology, vol. 41, no. 10, pp. 16431646, 1991.

[58] G. Groppel, T. Kapitany, and C. Baumgartner, "Cluster analysis of clinical seizure semiology of psychogenic nonepileptic seizures," Epilepsia, vol. 41, no. 5, pp. 610-614, 2000.

[59] G. D. Cascino, "Video-EEG monitoring in adults," Epilepsia, vol. 43, supplement 3, pp. 80-93, 2002.

[60] H. Yoshinaga, J. Hattori, H. Ohta et al., "Utility of the scalprecorded ictal EEG in childhood epilepsy," Epilepsia, vol. 42, no. 6, pp. 772-777, 2001.

[61] N. Desai, M. Shelke, A. Hegde, and K. N. Shah, "Role of short duration, outpatient video EEG monitoring in children," Neurology Asia, vol. 12, supplement 1, pp. 80-81, 2007.
[62] D. S. Goodin, M. J. Aminoff, and K. D. Laxer, "Detection of epileptiform activity by different noninvasive EEG methods in complex partial epilepsy," Annals of Neurology, vol. 27, no. 3, pp. 330-334, 1990.

[63] M. Salinsky, R. Kanter, and R. M. Dasheiff, "Effectiveness of multiple EEGs in supporting the diagnosis of epilepsy: an operational curve," Epilepsia, vol. 28, no. 4, pp. 331-334, 1987.

[64] D. S. Goodin and M. J. Aminoff, "Does the interictal EEG have a role in the diagnosis of epilepsy?" The Lancet, vol. 323, no. 8381, pp. 837-839, 1984.

[65] T. S. Walczak, M. L. Scheuer, S. Resor, and T. Pedley, "Prevalence and features of epilepsy without interictal epileptiform discharges," Neurology, vol. 43, supplement, pp. 287-288, 1993.

[66] S. Shinnar, A. T. Berg, S. L. Moshe et al., "Risk of seizure recurrence following a first unprovoked seizure in childhood: a prospective study," Pediatrics, vol. 85, no. 6, pp. 1076-1085, 1990.

[67] N. Callaghan, A. Garrett, and T. Coggin, "Withdrawal of anticonvulsant drugs in patients free of seizures for two years: a prospective study," The New England Journal of Medicine, vol. 318, no. 15, pp. 942-946, 1988, Erratum in The New England Journal of Medicine, vol. 319, no. 3, p. 188, 1988.

[68] C. Ajmone-Marsan and L. S. Zivin, "Factors related to the occurrence of typical paroxysmal abnormalities in the EEG records of epileptic patients," Epilepsia, vol. 11, no. 4, pp. 361-381, 1970.

[69] M. Sundaram, T. Hogan, M. Hiscock, and N. Pillay, "Factors affecting interictal spike discharges in adults with epilepsy," Electroencephalography and Clinical Neurophysiology, vol. 75, no. 4, pp. 358-360, 1990.

[70] J. Gotman, "Automatic detection of seizures and spikes," Journal of Clinical Neurophysiology, vol. 16, no. 2, pp. 130140, 1999.

[71] J. Gotman and M. G. Marciani, "Electroencephalographic spiking activity, drug levels, and seizure occurrence in epileptic patients," Annals of Neurology, vol. 17, no. 6, pp. 597-603, 1985.

[72] D. Schmidt, "The influence of antiepileptic drugs on the electroencephalogram: a review of controlled clinical studies," Electroencephalography and Clinical Neurophysiology, vol. 36, pp. 453-466, 1982.

[73] C. W. Bazil and T. A. Pedley, "General principles. Neurophysiological effects of antiepileptic drugs," in Antiepileptic Drugs, R. H. Levy, R. H. Mattson, B. S. Meldrum et al., Eds., pp. 23-35, Lippincott Williams \& Wilkins, New York, NY, USA, 2002.

[74] M. Adjouadi, D. Sanchez, M. Cabrerizo et al., "Interictal spike detection using the walsh transform," IEEE Transactions on Biomedical Engineering, vol. 51, no. 5, pp. 868-872, 2004.

[75] H. S. Liu, T. Zhang, and F. S. Yang, "A multistage, multimethod approach for automatic detection and classification of epileptiform EEG," IEEE Transactions on Biomedical Engineering, vol. 49, no. 12, pp. 1557-1566, 2002.

[76] D. R. Bennett, "Spike-wave complexes in normal flying personnel," Aerospace Medicine, vol. 38, no. 12, pp. 12761282, 1967.

[77] L. Zivin and C. Ajmone-Marsan, "Incidence and prognostic significance of epileptiform activity in the EEG of nonepileptic subjects," Brain, vol. 91, no. 4, pp. 751-778, 1968.

[78] G. B. Cavazzuti, "Epidemiology of different types of epilepsy in school age children of modena, Italy," Epilepsia, vol. 21, no. 1, pp. 57-62, 1980. 
[79] S. Shinnar, A. T. Berg, S. L. Moshe et al., "The risk of seizure recurrence after a first unprovoked afebrile seizure in childhood: an extended follow-up," Pediatrics, vol. 98, no. 2, pp. 216-225, 1996.

[80] D. L. Gilbert and C. R. Buncher, "An EEG should not be obtained routinely after first unprovoked seizure in childhood," Neurology, vol. 54, no. 3, pp. 635-641, 2000.

[81] A. Schreiner and B. Pohlmann-Eden, "Value of the early electroencephalogram after a first unprovoked seizure," Clinical EEG, vol. 34, no. 3, pp. 140-144, 2003.

[82] G. Barton, E. Hicks, V. H. Patterson et al., "Randomised study of antiepileptic drug withdrawal in patients in remission," The Lancet, vol. 337, no. 8751, pp. 1175-1180, 1991.

[83] S. Shinnar, E. P. Vining, E. D. Mellits et al., "Discontinuing antiepileptic medication in children with epilepsy after two years without seizures: a prospective study," The New England Journal of Medicine, vol. 313, no. 16, pp. 976-980, 1985.

[84] P. Tinuper, P. Avoni, R. Riva, F. Provini, E. Lugaresi, and A. Baruzzi, "The prognostic value of the electroencephalogram in antiepileptic drug withdrawal in partial epilepsies," Neurology, vol. 47, no. 1, pp. 76-78, 1996.

[85] Y. Schiller, G. D. Cascino, E. L. So, and R. R. Marsh, "Discontinuation of antiepileptic drugs after successful epilepsy surgery," Neurology, vol. 54, no. 2, pp. 346-349, 2000.

[86] C. Rathore, S. P. Sarma, and K. Radhakrishnan, "Prognostic importance of serial postoperative EEGs after anterior temporal lobectomy," Neurology, vol. 76, no. 22, pp. 1925-1931, 2011.

[87] L. E. Jeha, I. M. Najm, W. E. Bingaman et al., "Predictors of outcome after temporal lobectomy for the treatment of intractable epilepsy," Neurology, vol. 66, no. 12, pp. 19381940, 2006.

[88] S. S. Spencer, A. T. Berg, B. G. Vickrey et al., "Predicting longterm seizure outcome after resective epilepsy surgery. The multicenter study," Neurology, vol. 65, no. 6, pp. 912-918, 2005.

[89] M. Kaibara and W. T. Blume, "The postictal electroencephalogram," Electroencephalography and Clinical Neurophysiology, vol. 70, no. 2, pp. 99-104, 1988.

[90] M. Koutroumanidis, C. Martin-Miguel, M. J. Hennessy et al., "Interictal temporal delta activity in temporal lobe epilepsy: correlations with pathology and outcome," Epilepsia, vol. 45, no. 11, pp. 1351-1367, 2004.

[91] W. T. Blume, J. L. Borghesi, and J. F. Lemieux, "Interictal indices of temporal seizure origin," Annals of Neurology, vol. 34, no. 5, pp. 703-709, 1993.

[92] N. So, P. Gloor, L. F. Quesney, M. Jones-Gotman, A. Olivier, and F. Andermann, "Depth electrode investigations in patients with bitemporal epileptiform abnormalities," Annals of Neurology, vol. 25, no. 5, pp. 423-431, 1989.

[93] J. D. Geyer, E. Bilir, R. E. Faught, R. Kuzniecky, and F. Gilliam, "Significance of interictal temporal lobe delta activity for localization of the primary epileptogenic region," Neurology, vol. 52, no. 1, pp. 202-205, 1999.

[94] A. Gambardella, J. Gotman, F. Cendes, and F. Andermann, "Focal intermittent delta activity in patients with mesiotemporal atrophy: a reliable marker of the epileptogenic focus," Epilepsia, vol. 36, no. 2, pp. 122-129, 1995.

[95] G. Di Gennaro, P. P. Quarato, P. Onorati et al., "Localizing significance of temporal intermittent rhythmic delta activity (TIRDA) in drug-resistant focal epilepsy," Clinical Neurophysiology, vol. 114, no. 1, pp. 70-78, 2003.
[96] A. Ebner and M. Hoppe, "Noninvasive electroencephalography and mesial temporal sclerosis," Journal of Clinical Neurophysiology, vol. 12, no. 1, pp. 23-31, 1995.

[97] J. Engel Jr., "Recent advances in surgical treatment of temporal lobe epilepsy," Acta Neurologica Scandinavica, Supplement, vol. 86, no. 140, pp. 71-80, 1992.

[98] P. D. Williamson, J. A. French, V. M. Thadani et al., "Characteristics of medial temporal lobe epilepsy. II. Interictal and ictal scalp electroencephalography, neuropsychological testing, neuroimaging, surgical results, and pathology," Annals of Neurology, vol. 34, no. 6, pp. 781-787, 1993.

[99] R. M. Sadler, J. F. Lemieux, and W. T. Blume, "Potential fields of anterior temporal spikes," Electroencephalography and Clinical Neurophysiology, vol. 58, pp. 47-48, 1984.

[100] J. S. Ebersole and P. B. Wade, "Spike voltage topography identifies two types of frontotemporal epileptic foci," Neurology, vol. 41, no. 9, pp. 1425-1433, 1991.

[101] B. A. Malow, R. Kushwaha, X. Lin, K. J. Morton, and M. S. Aldrich, "Relationship of interictal epileptiform discharges to sleep depth in partial epilepsy," Electroencephalography and Clinical Neurophysiology, vol. 102, no. 1, pp. 20-26, 1997.

[102] M. Sammaritano, G. L. Gigli, and J. Gotman, "Interictal spiking during wakefulness and sleep and the localization of foci in temporal lobe epilepsy," Neurology, vol. 41, no. 2 I, pp. 290-297, 1991.

[103] M. D. Holmes, C. B. Dodrill, A. J. Wilensky, L. M. Ojemann, and G. A. Ojemann, "Unilateral focal preponderance of interictal epileptiform discharges as a predictor of seizure origin," Archives of Neurology, vol. 53, no. 3, pp. 228-232, 1996.

[104] F. Cendes, L. M. Li, C. Watson, F. Andermann, F. Dubeau, and D. L. Arnold, "Is ictal recording mandatory in temporal lobe epilepsy? Not when the interictal electroencephalogram and hippocampal atrophy coincide," Archives of Neurology, vol. 57, no. 4, pp. 497-500, 2000.

[105] J. Engel, S. Wiebe, J. French et al., "Practice parameter: temporal lobe and localized neocortical resections for epilepsy," Neurology, vol. 60, no. 4, pp. 538-547, 2003.

[106] J. I. Sirven, J. D. Liporace, J. A. French, M. J. O’Connor, and M. R. Sperling, "Seizures in temporal lobe epilepsy. I. reliability of scalp/sphenoidal ictal recording," Neurology, vol. 48, no. 4, pp. 1041-1046, 1997.

[107] N. So, A. Olivier, F. Andermann, P. Gloor, and L. F. Quesney, "Results of surgical treatment in patients with bitemporal epileptiform abnormalities," Annals of Neurology, vol. 25, no. 5, pp. 432-439, 1989.

[108] H. Gastaut, "So-called "psychomotor" and temporal epilepsy-a critical study," Epilepsia, vol. 7, pp. 85-138, 1953.

[109] G. D. Casino, M. R. Trenerry, E. L. So et al., "Routine EEG and temporal lobe epilepsy: relation to long term EEG monitoring, quantitative MRI, and operative outcome," Epilepsia, vol. 37, no. 7, pp. 651-656, 1995.

[110] D. W. Klass, "Electroencephalographic manifestations of complex partial seizures," in Complex Partial Seizures and their Treatment, J. K. Penry and D. D. Daly, Eds., pp. 113-140, Raven, New York, NY, USA, 1975.

[111] W. T. Blume, G. B. Young, and J. F. Lemieux, "EEG morphology of partial epileptic seizures," Electroencephalography and Clinical Neurophysiology, vol. 57, no. 4, pp. 295-302, 1984.

[112] W. T. Blume and M. Kaibara, "The start-stop-start phenomenon of subdurally recorded seizures," Electroencephalography and Clinical Neurophysiology, vol. 86, no. 2, pp. 9499, 1993. 
[113] N. Atalla, B. Abou-Khalil, and T. Fakhoury, "The startstop-start phenomenon in scalp-sphenoidal ictal recordings," Electroencephalography and Clinical Neurophysiology, vol. 98, no. 1, pp. 9-13, 1996.

[114] M. W. Risinger, J. Engel Jr., P. C. Van Ness, T. R. Henry, and P. H. Crandall, "Ictal localization of temporal lobe seizures with scalp/sphenoidal recordings," Neurology, vol. 39, no. 10, pp. 1288-1293, 1989.

[115] B. J. Steinhoff, N. K. So, S. Lim, and H. O. Luders, "Ictal scalp EEG in temporal lobe epilepsy with unitemporal versus bitemporal interictal epileptiform discharges," Neurology, vol. 45, no. 5, pp. 889-896, 1995.

[116] T. S. Walczak, R. A. Radtke, and D. V. Lewis, "Accuracy and interobserver reliability of scalp ictal EEG," Neurology, vol. 42, no. 12, pp. 2279-2285, 1992.

[117] J. S. Ebersole and S. V. Pacia, "Localization of temporal lobe foci by ictal EEG patterns," Epilepsia, vol. 37, no. 4, pp. 386399, 1996.

[118] A. Gil-Nagel and M. W. Risinger, "Ictal semiology in hippocampal versus extrahippocampal temporal lobe epilepsy," Brain, vol. 120, no. 1, pp. 183-192, 1997.

[119] N. Foldvary, N. Lee, G. Thwaites et al., "Clinical and electrographic manifestations of lesional neocortical temporal lobe epilepsy," Neurology, vol. 49, no. 3, pp. 757-763, 1997.

[120] D. W. King and C. Ajmone-Marsan, "Clinical features and ictal patterns in epileptic patients with EEG temporal lobe foci," Annals of Neurology, vol. 2, pp. 138-147, 1977.

[121] W. H. Theodore, R. J. Porter, and J. K. Penry, "Complex partial seizures: clinical characteristics and differential diagnosis," Neurology, vol. 33, no. 9, pp. 1115-1121, 1983.

[122] L. F. Quesney, M. W. Risinger, and D. A. Shewmon, "Extracranial EEG evaluation," in Surgical Treatment of the Epilepsies, J. Engel Jr., Ed., pp. 173-195, Raven, New York, NY, USA, 2nd edition, 1993.

[123] C. Barba, G. Barbati, L. Minotti, D. Hoffmann, and P. Kahane, "Ictal clinical and scalp-EEG findings differentiating temporal lobe epilepsies from temporal "plus" epilepsies," Brain, vol. 130, no. 7, pp. 1957-1967, 2007.

[124] R. Schulz, H. O. Lüders, M. Hoppe, I. Tuxhorn, T. May, and A. Ebner, "Interictal EEG and ictal scalp EEG propagation are highly predictive of surgical outcome in mesial temporal lobe epilepsy," Epilepsia, vol. 41, no. 5, pp. 564-570, 2000.

[125] Y. Aghakhani, A. Rosati, F. Dubeau, A. Olivier, and F. Andermann, "Patients with temporoparietal ictal symptoms and inferomesial EEG do not benefit from anterior temporal resection," Epilepsia, vol. 45, no. 3, pp. 230-236, 2004.

[126] P. D. Williamson, D. D. Spencer, S. S. Spencer, R. A. Novelly, and R. H. Mattson, "Complex partial seizures of frontal lobe origin,” Annals of Neurology, vol. 18, no. 4, pp. 497-504, 1985.

[127] P. D. Williamson, V. M. Thadani, T. M. Darcey, D. D. Spencer, S. S. Spencer, and R. H. Mattson, "Occipital lobe epilepsy: clinical characteristics, seizure spread patterns, and results of surgery," Annals of Neurology, vol. 31, no. 1, pp. 3-13, 1992.

[128] P. D. Williamson, P. A. Boon, V. M. Thadani et al., "Parietal lobe epilepsy: diagnostic considerations and results of surgery," Annals of Neurology, vol. 31, no. 2, pp. 193-201, 1992.

[129] R. C. Schneider, E. C. Crosby, and S. M. Farhar, "Extratemporal lesions triggering the temporal-lobe syndrome: the role of association bundles," Journal of Neurosurgery, vol. 22, pp. 246-263, 1964.

[130] S. Spencer, P. Williamson, and S. Bridgers, "Reliability and accuracy of localization by scalp ictal EEG," Neurology, vol. 35, no. 11, pp. 1567-1575, 1985.
[131] J. Gotman and L. Y. Wang, "State dependent spike detection: validation," Electroencephalography and Clinical Neurophysiology, vol. 83, no. 1, pp. 12-18, 1992.

[132] N. S. O’Neill, M. Javidan, and Z. J. Koles, "Identification of the temporal components of seizure onset in the scalp EEG," Canadian Journal of Neurological Sciences, vol. 28, no. 3, pp. 245-253, 2001.

[133] S. M. Haas, M. G. Frei, and I. Osorio, "Strategies for adapting automated seizure detection algorithms," Medical Engineering \& Physics, vol. 29, no. 8, pp. 895-909, 2007.

[134] C. E. D’Attellis, S. I. Isaacson, and R. O. Sirne, "Detection of epileptic events in electroencephalograms using wavelet analysis," Annals of Biomedical Engineering, vol. 25, no. 2, pp. 286-293, 1997.

[135] A. S. Zandi, M. Javidan, G. A. Dumont, and R. Tafreshi, "Automated real-time epileptic seizure detection in scalp EEG recordings using an algorithm based on wavelet packet transform," IEEE Transactions on Biomedical Engineering, vol. 57, no. 7, pp. 1639-1651, 2010.

[136] J. Lieb, R. Dasheiff, and J. Engel, "Role of the frontal lobes in the propagation of mesial temporal lobe seizures," Epilepsia, vol. 32, no. 6, pp. 822-837, 1991.

[137] Y. Sakai, H. Nagano, A. Sakata et al., "Localization of epileptogenic zone in temporal lobe epilepsy by ictal scalp EEG," Seizure, vol. 11, no. 3, pp. 163-168, 2002.

[138] M. M. Jan, M. Sadler, and S. R. Rahey, "Lateralized postictal EEG delta predicts the side of seizure surgery in temporal lobe epilepsy," Epilepsia, vol. 42, no. 3, pp. 402-405, 2001.

[139] D. King and S. Spencer, "Invasive electroencephalography in mesial temporal lobe epilepsy," Journal of Clinical Neurophysiology, vol. 12, no. 1, pp. 32-45, 1995.

[140] F. Dubeau and R. S. McLachlan, "Invasive electrographic recording techniques in temporal lobe epilepsy," Canadian Journal of Neurological Sciences, vol. 27, no. 1, pp. S29-S34, 2000.

[141] F. Wendling, A. Hernandez, J. J. Bellanger, P. Chauvel, and F. Bartolomei, "Interictal to ictal transition in human temporal lobe epilepsy: insights from a computational model of intracerebral EEG," Journal of Clinical Neurophysiology, vol. 22, no. 5, pp. 343-356, 2005.

[142] W. Y. Jung, S. V. Pacia, and O. Devinsky, "Neocortical temporal lobe epilepsy: intracranial EEG features and surgical outcome," Journal of Clinical Neurophysiology, vol. 16, no. 5, pp. 419-425, 1999.

[143] M. Y. Chung, T. S. Walczak, D. V. Lewis, D. V. Dawson, and R. Radtke, "Temporal lobectomy and independent bitemporal interictal activity: what degree of lateralization is sufficient?" Epilepsia, vol. 32, no. 2, pp. 195-201, 1991.

[144] C. Munari, J. Talairach, A. Bonis, G. Szikla, and J. Bancaud, "Differential diagnosis between temporal and "perisylvian" epilepsy in a surgical perspective," Acta Neurochirurgica. Supplement, vol. 30, pp. 97-101, 1980.

[145] J. Isnard, M. Guenot, M. Sindou, and F. Mauguiere, "Clinical manifestations of insular lobe seizures: a stereoelectroencephalographic study," Epilepsia, vol. 45, no. 9, pp. 1079-1090, 2004.

[146] J. Rémi, C. Vollmar, A. de Marinis, J. Heinlin, A. Peraud, and S. Noachtar, "Congruence and discrepancy of interictal and ictal EEG with MRI lesions in focal epilepsies," Neurology, vol. 77, no. 14, pp. 1383-1390, 2011.

[147] S. V. Pacia and J. S. Ebersole, "Intracranial EEG substrates of scalp ictal patterns from temporal lobe foci," Epilepsia, vol. 38, no. 6, pp. 642-654, 1997. 
[148] D. G. Vossler, D. L. Kraemer, A. M. Haltiner et al., "Intracranial EEG in temporal lobe epilepsy: location of seizure onset relates to degree of hippocampal pathology," Epilepsia, vol. 45, no. 5, pp. 497-503, 2004.

[149] D. W. Seo and S. B. Hong, "Epileptogenic foci on subdural recording in intractable epilepsy patients with temporal dysembryoplastic neuroepithelial tumor," Journal of Korean Medical Science, vol. 18, no. 4, pp. 559-565, 2003.

[150] W. W. Sutherling and D. S. Barth, "Magnetoencephalography in clinical epilepsy studies: the UCLA experience," Advances in Neurology, vol. 54, pp. 231-245, 1990.

[151] R. Paetau, M. Kajola, J. Karhu et al., "Magnetoencephalographic localization of epileptic cortex-impact on surgical treatment," Annals of Neurology, vol. 32, no. 1, pp. 106-109, 1992.

[152] D. S. Eliashiv, S. M. Elsas, K. Squires, I. Fried, and J. Engel, "Ictal magnetic source imaging as a localizing tool in partial epilepsy," Neurology, vol. 59, no. 10, pp. 1600-1610, 2002.

[153] J. R. Smith, B. J. Schwartz, C. Gallen et al., "Utilization of multichannel magnetoencephalography in the guidance of ablative seizure surgery," Journal of Epilepsy, vol. 8, no. 2, pp. 119-130, 1995.

[154] J. S. Ebersole, K. C. Squires, S. D. Eliashiv, and J. R. Smith, "Application of magnetic source imaging in evaluation of candidates for epilepsy surgery," in Neuroimaging Clinic of North America, J. Kucharczyk, T. P. L. Roberts, M. F. Mosley et al., Eds., vol. 5, part 2, pp. 267-288, WB Saunders, Philadelphia, Pa, USA, 1995.

[155] E. Pataraia, G. Lindinger, L. Deecke, D. Mayer, and C. Baumgartner, "Combined MEG/EEG analysis of the interictal spike complex in mesial temporal lobe epilepsy," Neuroimage, vol. 24, no. 3, pp. 607-614, 2005. 


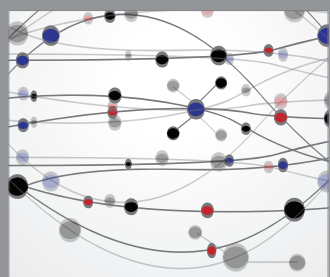

The Scientific World Journal
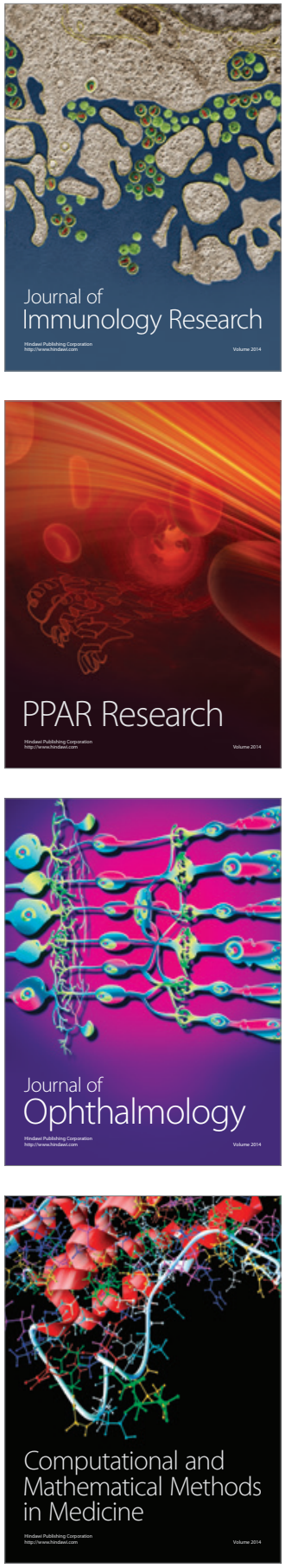

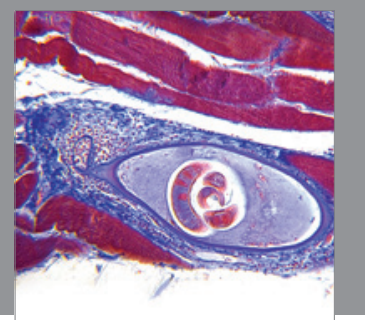

Gastroenterology

Research and Practice
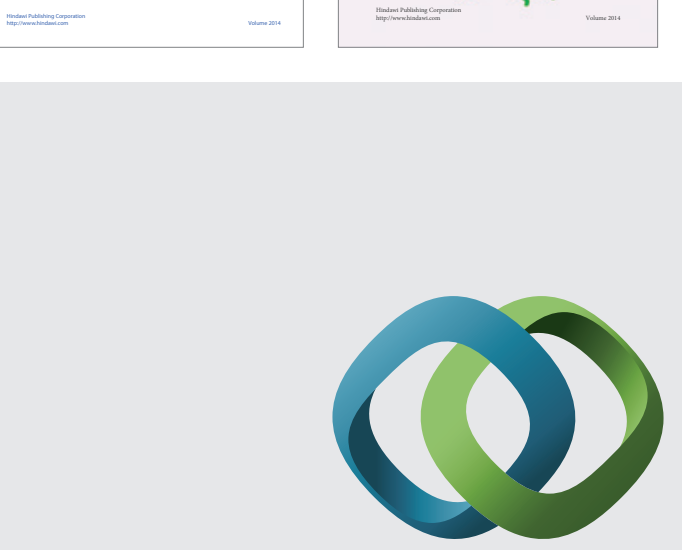

\section{Hindawi}

Submit your manuscripts at

http://www.hindawi.com
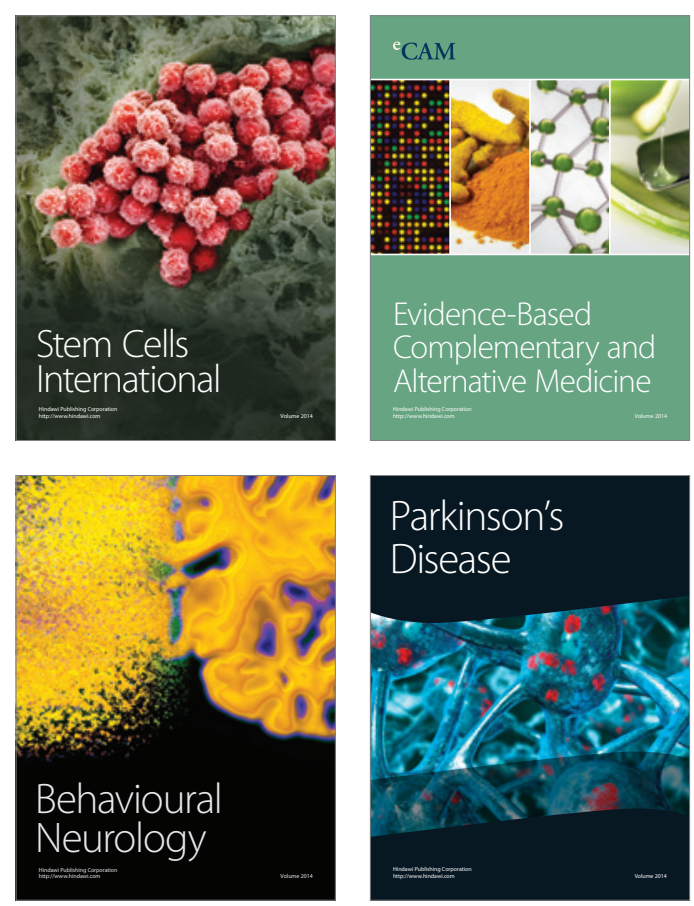



Journal of
Diabetes Research

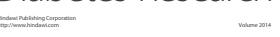



Disease Markers
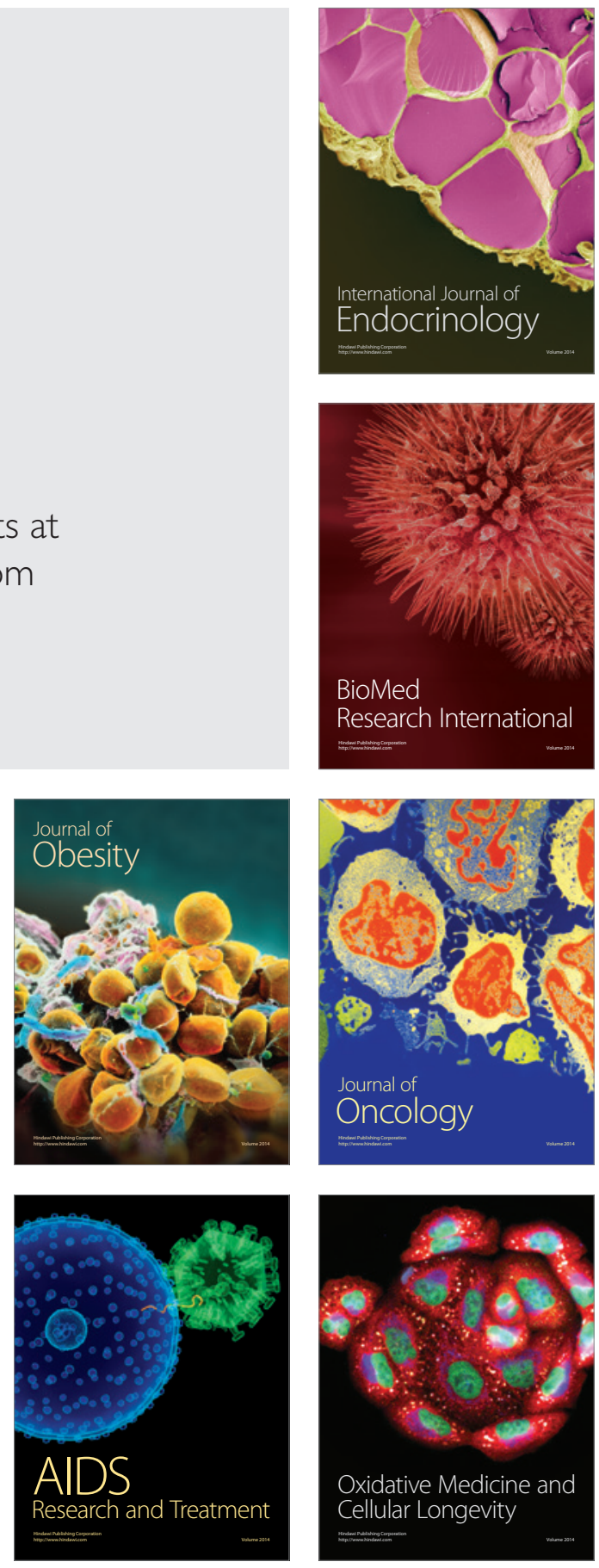